\title{
Estimates on the Bergman Kernels in a Tangential Direction on Pseudoconvex Domains in $\mathbb{C}^{3}$
}

\author{
Sanghyun Cho \\ Department of Mathematics, Sogang University, Seoul 04107, Republic of Korea \\ Correspondence should be addressed to Sanghyun Cho; shcho@sogang.ac.kr
}

Received 2 March 2018; Accepted 5 July 2018; Published 18 July 2018

Academic Editor: Milan Pokorny

Copyright (C) 2018 Sanghyun Cho. This is an open access article distributed under the Creative Commons Attribution License, which permits unrestricted use, distribution, and reproduction in any medium, provided the original work is properly cited.

Let $\Omega$ be a smoothly bounded pseudoconvex domain in $\mathbb{C}^{3}$ and assume that $T_{\Omega}^{\text {reg }}\left(z_{0}\right)<\infty$ where $z_{0} \in b \Omega$, the boundary of $\Omega$. Then we get optimal estimates of the Bergman kernel function along some "almost tangential curve" $C_{b}\left(z_{0}, \delta_{0}\right) \subset \Omega \cup\left\{z_{0}\right\}$.

\section{Introduction}

Let $\Omega$ be a bounded domain in $\mathbb{C}^{n}$. A natural operator on $\Omega$ is the orthogonal projection

$$
P: L^{2}(\Omega) \longrightarrow H(\Omega) \cap L^{2}(\Omega),
$$

where $H(\Omega)$ denotes the holomorphic functions on $\Omega$. There is a corresponding kernel function $K_{\Omega}(z, w)$, called the Bergman kernel function on $\Omega$. The nature of the singularity of $K_{\Omega}(z, w)$ tells us much about the holomorphic function theory of the domain in question and has been studied extensively since Bergman's original inquiries [1].

One of the methods for the estimates of the Bergman kernel is to construct maximal size of polydiscs in $\Omega$ where we have a plurisubharmonic function with maximal Hessian. For strongly pseudoconvex domains in $\mathbb{C}^{n}$, these polydiscs are of size $\delta>0$ in normal direction and of size $\delta^{1 / 2}$ in tangential directions. For weakly pseudoconvex domains, the size of the polydisc in tangential directions depends on the boundary geometry of $\Omega$ near $z_{0} \in b \Omega$, and hence we need complete analysis of the boundary geometry near $z_{0}$.

However these analyses and hence the optimal estimates on the Bergman kernels are done only for special type of pseudoconvex domains of finite type in $\mathbb{C}^{n}$. These domains are, for example, pseudoconvex domains of finite type in $\mathbb{C}^{2}$ [2-4], decoupled, convex, or uniformly extendable domains of finite type in $\mathbb{C}^{n}[5-7]$, or pseudoconvex domains in $\mathbb{C}^{n}$ with $(n-2)$ positive eigenvalues $[8,9]$. For the estimates for weighted Bergman projections, one can also refer to [10-12].
Nevertheless, the optimal estimates for general pseudoconvex domains of finite type in $\mathbb{C}^{n}, n>2$, are not known, even for $n=3$ case.

Assume that $\Omega$ is a smoothly bounded domain in $\mathbb{C}^{n}$ with smooth defining function $r$ with smooth boundary, $b \Omega$. Regular finite 1-type at $z_{0} \in b \Omega$, denoted by $T_{\Omega}^{r e g}\left(z_{0}\right)$, is the maximum order of vanishing of $r \circ \gamma$ for all one complex dimensional regular curve $\gamma, \gamma(0)=z_{0}$, and $\gamma^{\prime}(0) \neq 0$. Thus $T_{\Omega}^{r e g}\left(z_{0}\right)$ satisfies

$$
\Delta_{n-1}\left(z_{0}\right) \leq T_{\Omega}^{r e g}\left(z_{0}\right) \leq \Delta_{1}\left(z_{0}\right),
$$

where $\Delta_{q}\left(z_{0}\right), 1 \leq q \leq n-1$, denotes finite $q$-type in the sense of D'Angelo [13]. Note that $\Delta_{n-1}\left(z_{0}\right)=T_{B G}\left(z_{0}\right)$ where $T_{B G}\left(z_{0}\right)$ is the type in the sense of Bloom-Graham.

Remark 1. Consider the domain $\Omega$ [13] in $\mathbb{C}^{3}$ defined by

$$
r(z)=\operatorname{Re} z_{3}+\left|z_{1}^{2}-z_{2}^{3}\right|^{2}
$$

Then $T_{\Omega}^{r e g}(0)=6$ and $\Delta_{2}(0)=4$ while $\Delta_{1}(0)=\infty$ as the complex analytic curve $\gamma(t)=\left(t^{3}, t^{2}, 0\right)$ lies in the boundary. Note that $\gamma(t)$ is not regular curve.

In the sequel, we let $\Omega$ be a smoothly bounded pseudoconvex domain in $\mathbb{C}^{3}$, and assume that $T_{\Omega}^{r e g}\left(z_{0}\right)=\eta<\infty$ where $z_{0} \in b \Omega$. Let $C_{b}\left(z_{0}, \delta_{0}\right) \subset \Omega \cup\left\{z_{0}\right\}$ be the "almost tangential curve" connecting a point $z^{\delta_{0}} \in \Omega$ and $z_{0} \in b \Omega$ as defined in (20). Note that $\operatorname{dist}\left(z^{\delta}, b \Omega\right) \approx \delta$ for each $z^{\delta} \epsilon$ 
$C_{b}\left(z_{0}, \delta_{0}\right)$. Set $\tau_{1}=\delta^{1 / \eta}, \tau_{2}=\tau\left(z^{\delta}, \delta\right)$ where $\tau\left(z^{\delta}, \delta\right)$ is defined in (51).

Theorem 2. Let $\Omega$ be a smoothly bounded pseudoconvex domain in $\mathbb{C}^{3}$ and assume that $T_{\Omega}^{r e g}\left(z_{0}\right)<\infty$ where $z_{0} \in b \Omega$. Then $K_{\Omega}\left(z^{\delta}, z^{\delta}\right)$, the Bergman kernel function of $\Omega$ at $z^{\delta} \in$ $C_{b}\left(z_{0}, \delta_{0}\right)$, satisfies

$$
K_{\Omega}\left(z^{\delta}, z^{\delta}\right) \approx \delta^{-2} \tau_{1}^{-2} \tau_{2}^{-2}
$$

Theorem 3. Let $\Omega$ and $z_{0} \in b \Omega$ be as in Theorem 2. For each $\alpha=\left(\alpha_{1}, \alpha_{2}, \alpha_{3}\right)$, there is a constant $C_{\alpha}>0$, independent of $\delta>0$, such that

$$
\left|D_{z}^{\alpha} K_{\Omega}\left(z, z^{\delta}\right)\right| \leq C_{\alpha} \delta^{-2-\alpha_{3}} \tau_{1}^{-2-\alpha_{1}} \tau_{2}^{-2-\alpha_{2}},
$$

for $z \in \Omega$ and $z^{\delta} \in C_{b}\left(z_{0}, \delta_{0}\right)$.

Remark 4. (1) In Theorems 2 and 3, we do not assume that $\Delta_{1}\left(z_{0}\right)<\infty$, but we assume only that $T_{\Omega}^{r e g}\left(z_{0}\right)<\infty$ (see Remark 1). With this weaker condition, we get optimal estimates for Bergman kernel function along special "almost tangential" direction, $C_{b}\left(z_{0}, \delta_{0}\right)$, but not normal or arbitrary direction.

(2) In [14], Herbort gives an example of a domain $\Omega_{H} \subset$ $\mathbb{C}^{3}$ where the Bergman kernel grows logarithmically when $z \in \Omega$ approaches to $z_{0} \in b \Omega$ in normal direction. Set

$$
\begin{aligned}
& \Omega_{H} \\
& \quad=\left\{\left.z \in \mathbb{C}^{3}\left|\operatorname{Re} z_{3}+\right| z_{1}\right|^{6}+\left|z_{1}\right|^{2}\left|z_{2}\right|^{2}+\left|z_{2}\right|^{6}<0\right\},
\end{aligned}
$$

and for each small $\delta>0$, set $z^{\delta}=(0,0,-\delta)$. Thus $z^{\delta}$ approaches to $0 \in b \Omega_{H}$ in normal direction as $\delta \longrightarrow 0$. In this case, Herbort shows that $K_{\Omega}\left(z^{\delta}, z^{\delta}\right) \approx \delta^{-3}(-\log \delta)^{-1}$; that is, the kernel grows logarithmically. For the same domain $\Omega_{H}$ in (6), we note that $\eta=T_{\Omega}^{r e g}\left(z_{0}\right)=6$ and hence $\tau_{1}=\delta^{1 / 6}$, $\tau_{2}=\delta^{1 / 3}$ in (4). Set $C_{b}\left(z_{0}, \delta_{0}\right):=\left\{\left(\delta^{1 / 6} / 2,0,-\delta\right): 0 \leq \delta \leq\right.$ $\left.\delta_{0}\right\}$. Then $z^{\delta}:=\left(\delta^{1 / 6} / 2,0,-\delta\right) \in C_{b}\left(z_{0}, \delta_{0}\right)$ approaches to $0 \in b \Omega$ in "almost tangential direction". In the Appendix of this paper, we will show that

$$
K_{\Omega_{H}}\left(z^{\delta}, z^{\delta}\right) \approx \delta^{-2} \tau_{1}^{-2} \tau_{2}^{-2}=\delta^{-3} .
$$

In Section 2, we will construct special coordinates which reflect the regular finite type condition, $\Delta_{2}\left(z_{0}\right) \leq T_{\Omega}^{r e g}\left(z_{0}\right)=$ $\eta<\infty$, and then show that $r(z)$ vanishes to order $\eta$ in $z_{1}$ direction. We then consider the slices of $\Omega$ by fixing $z_{1}$. Then the domains become domains in $\mathbb{C}^{2}$, and hence we can handle them. Also, the condition $\Delta_{2}\left(z_{0}\right)<\infty$ acts like the condition $\Delta_{1}\left(z_{0}\right)<\infty$ on these slices.

For the estimates of $K(z, w)$, Catlin [2, 15] constructed plurisubharmonic functions with maximal Hessian near each thin $\delta$-strip of $b \Omega$ (Section 3 of [2]). In this paper, however, we will construct these functions only on nonisotropic polydiscs $Q_{a \delta}\left(z^{\delta}\right) \subset \subset \Omega$ for each $z^{\delta} \in C_{b}\left(z_{0}, \delta_{0}\right)$ (Proposition 23 ). This avoids complicated technical parts in Section 3 of [2]. To get estimates of $K_{\Omega}\left(z, z^{\delta}\right), z \in \Omega, z^{\delta} \in C_{b}\left(z_{0}, \delta_{0}\right)$, we consider dilated domains $D_{\delta}$ for each $\delta>0$. Then the polydisc $Q_{a \delta}\left(z^{\delta}\right) \subset \subset \Omega$ becomes $P(0,1) \subset \subset D_{\delta}$, independent of $\delta>0$, where $P(0,1)$ is a polydisc of radius one with center at the origin. Therefore the uniform $1 / 2$-subelliptic estimates for $\bar{\partial}$-equation hold on $P(0,1)$, and the estimates for $K_{\Omega}\left(z, z^{\delta}\right)$ follow.

Remark 5. Let $\Omega$ be a smoothly bounded pseudoconvex domain in $\mathbb{C}^{3}$, and assume that $\Delta_{1}\left(z_{0}\right)<\infty$, where $z_{0} \in b \Omega$. Then the conditions of Theorems 2 and 3 are satisfied. Near future, using the results of Theorems 2 and 3, we hope we can prove some function theories on $\Omega$, for example, the existence of peak function for $\Omega$ that peaks at $z_{0} \in b \Omega$ or necessary conditions for the Hölder estimates for $\bar{\partial}$-equation.

\section{Special Coordinates}

In the sequel, we let $\Omega$ be a smoothly bounded pseudoconvex domain in $\mathbb{C}^{3}$ and assume that $m=\Delta_{2}\left(z_{0}\right) \leq \eta=T_{\Omega}^{r e g}\left(z_{0}\right)<$ $\infty, z_{0} \in b \Omega$. Note that $m$ and $\eta$ are positive integers. Without loss of generality, we may assume that $z_{0}=0$. In the sequel, we let $\alpha=\left(\alpha_{1}, \alpha_{2}, \alpha_{3}\right)$ and $\beta=\left(\beta_{1}, \beta_{2}, \beta_{3}\right)$ be multi-indices and set $\alpha^{\prime}=\left(\alpha_{1}, \alpha_{2}\right)$ and $z^{\prime}=\left(z_{1}, z_{2}\right)$, etc. In Theorem 3.1 in [16], You constructed special coordinates which represent the local geometry of $b \Omega$ near $z_{0}$.

Theorem 6. Let $\Omega$ be a smoothly bounded pseudoconvex domain in $\mathbb{C}^{3}$ with smooth defining function $r$ and assume $T_{\Omega}^{r e g}(0)=\eta<\infty, 0 \in b \Omega$. Then there is a holomorphic coordinate system $z=\left(z_{1}, z_{2}, z_{3}\right)$ about 0 such that

$$
\begin{gathered}
r(z)=\operatorname{Re} z_{3}+\sum_{\substack{\left|\alpha^{\prime}\right|+\left|\beta^{\prime}\right|=m \\
\left|\alpha^{\prime}\right|,\left|\beta^{\prime}\right|>0}}^{\eta} a_{\alpha^{\prime}, \beta^{\prime}} z^{\alpha^{\prime}} \bar{z}^{\beta^{\prime}} \\
+\mathcal{O}\left(\left|z_{3}\right||z|+\left|z^{\prime}\right|^{\eta+1}\right),
\end{gathered}
$$

(2) $|r(t, 0,0)| \approx|t|^{\eta}$,

where

$$
a_{\alpha^{\prime}, \beta^{\prime}} \neq 0
$$

with $\alpha_{2}+\beta_{2}=m$ for some $\alpha_{2}>0, \beta_{2}>0$.

(Idea of the proof) by the standard holomorphic coordinate changes, $r(w)$ has the Taylor series expansion as in (8). Since $T_{\Omega}^{r e g}(0)=\eta$, there is a regular curve which we may assume that $\gamma(t)=\left(t, \gamma_{2}(t), \mathcal{O}\left(t^{\eta}\right)\right)$ satisfying $|r(\gamma(t))| \approx|t|^{\eta}$ for all sufficiently small $t \in \mathbb{C}$. Set $z=\left(w_{1}, w_{2}+\gamma_{2}\left(w_{1}\right), w_{3}\right)$. Then, in $z$ coordinates, $r(z)$ has representation satisfying (8). Also (9) follows from the condition that $m=\Delta_{2}(0)$.

Remark 7. (1) The second condition in (8) and property (9) say that $r(z)$ vanishes to order $\eta$ along $z_{1}$ axis and order $m$ along $z_{2}$ axis.

(2) There are much more terms (mixed with $z_{1}, z_{2}$ and their conjugates), compared to the $h$-extensible domain cases, in the summation part of (8). 
In conjunction with multitype $\mathscr{M}(0)=\left(1, m, m_{3}\right)$, we need to consider the dominating terms (in size) among the mixed terms in $z_{1}$ and $z_{2}$ variables in the summation part of (8). Using the notations of Section 3.2 in [16], set

$$
\begin{aligned}
\Gamma & =\left\{\left(\alpha^{\prime}, \beta^{\prime}\right) ; a_{\alpha^{\prime}, \beta^{\prime}} \neq 0, m \leq\left|\alpha^{\prime}\right|+\left|\beta^{\prime}\right|\right. \\
& \left.\leq \eta, \text { and }\left|\alpha^{\prime}\right|,\left|\beta^{\prime}\right|>0\right\} \\
S & =\left\{(p, q) ; \alpha_{1}+\beta_{1}=p, \alpha_{2}+\beta_{2}\right. \\
& \left.=q \text { for some }\left(\alpha^{\prime}, \beta^{\prime}\right) \in \Gamma\right\} \cup\{(\eta, 0)\} .
\end{aligned}
$$

Then there are $\left(p_{v}, q_{v}\right) \in S$ for $v=0,1, \ldots, N$ and $\eta_{v}, \lambda_{v}>0$ for $v=1, \ldots, N$, such that

$$
\text { (1) } \begin{aligned}
\left(p_{0}, q_{0}\right) & =(\eta, 0),\left(p_{N}, q_{N}\right)=(0, m), \lambda_{N} \\
& =m, \eta_{1}=\eta \\
\text { (2) } p_{0} & >p_{1}>\ldots>p_{N} \text { and } \\
q_{0} & <q_{1}<\ldots<q_{N} \\
\text { (3) } \lambda_{1} & <\lambda_{2}<\ldots<\lambda_{N} \text { and } \\
\eta_{1} & >\eta_{2}>\ldots>\eta_{N}
\end{aligned}
$$

(4) $\frac{p_{v-1}}{\eta_{v}}+\frac{q_{v-1}}{\lambda_{v}}=1$ and

$$
\frac{p_{v}}{\eta_{v}}+\frac{q_{v}}{\lambda_{v}}=1
$$

(5) $a_{\alpha^{\prime}, \beta^{\prime}}=0$

$$
\text { if } \frac{\alpha_{1}+\beta_{1}}{\eta_{v}}+\frac{\alpha_{2}+\beta_{2}}{\lambda_{v}}<1 \text { for each } v=1, \ldots, N \text {. }
$$

Remark 8. (1) Here, $p_{\nu}$ 's and $q_{\nu}$ 's are the exponents of $z_{1}$ and $z_{2}$, respectively, in the dominating terms in the summation part of (8).

(2) If $\Delta_{1}\left(z_{0}\right)<\infty$, then the expression in (8) will be similar to that of $\mathbb{C}^{2}$ case in [2], and hence we need not consider the above complicated pairs.

Set $t_{0}=\eta$. If $1 \leq k \leq m$, then $q_{v-1}<k \leq q_{\nu}$ for some $v=1, \ldots, N$. In this case, set

$$
t_{k}=\eta_{v}\left(1-\frac{k}{\lambda_{v}}\right), \quad \text { i.e., } \frac{t_{k}}{\eta_{v}}+\frac{k}{\lambda_{v}}=1 .
$$

Then $\left(p_{v-1}, q_{v-1}\right),\left(t_{l}, l\right)$, and $\left(p_{v}, q_{v}\right)$ are colinear points in the first quadrant of the plane, and $\lambda_{\nu}$ (resp., $\eta_{\nu}$ ) is the intercept of $q$-axis (resp., $p$-axis) of this line. Let $L_{\nu}$ be the line segment from $\left(p_{\nu-1}, q_{\nu-1}\right)$ to $\left(p_{v}, q_{v}\right)$ for $v=1, \ldots, N$, set $L=L_{1} \cup L_{2} \cup$ $\ldots \cup L_{N}, \Gamma_{L}=\left\{\left(\alpha^{\prime}, \beta^{\prime}\right) \in \Gamma ;\left(\alpha_{1}+\beta_{1}, \alpha_{2}+\beta_{2}\right) \in L\right\}$, and set

$$
\begin{aligned}
\Lambda & =\left\{\left(\alpha^{\prime}, \beta^{\prime}\right) \in \Gamma_{L} ; \alpha^{\prime}+\beta^{\prime}=\left(p_{v}, q_{v}\right), \alpha_{2}>0, \beta_{2}\right. \\
& >0, \quad v=1, \ldots, N\} .
\end{aligned}
$$

As in Corollary 3.8 and Remark 3.9 in [16], we can rewrite (8) so that

$$
\begin{aligned}
& r(z) \\
& =\operatorname{Re} z_{3}+\sum_{\Gamma_{L}-\Lambda} a_{\alpha, \beta} z^{\prime \alpha} \bar{z}^{\prime \beta} \\
& \quad+\sum_{\nu=1}^{N} \sum_{\substack{\alpha_{2}+\beta_{2}=q_{\nu} \\
\alpha_{2}>0, \beta_{2}>0}} M_{\alpha_{2}, \beta_{2}}^{v}\left(z_{1}\right) z_{2}^{\alpha_{2}} \bar{z}_{2}^{\beta_{2}} \\
& +\mathcal{O}\left(\left|z_{3}\right||z|+\sum_{\nu=1}^{N} \sum_{l=q_{v-1}}^{q_{v}}\left|z_{1}\right|^{\left[t_{l}\right]+1}\left|z_{2}\right|^{l}+\left|z_{2}\right|^{m+1}\right),
\end{aligned}
$$

where $M_{\alpha_{2}, \beta_{2}}^{v}\left(z_{1}\right)$ is a nontrivial homogeneous polynomial of degree $p_{\nu}$ given by

$$
M_{\alpha_{2}, \beta_{2}}^{v}\left(z_{1}\right)=\sum_{\alpha_{1}+\beta_{1}=p_{v}} a_{\alpha_{1}, \beta_{1}} z_{1}^{\alpha_{1}} \bar{z}_{1}^{\beta_{1}},
$$

and there are a small constant $a_{0}>0$, and $d \in\left\{z_{1} \in \mathbb{C} ;\left|z_{1}\right|=\right.$ $1\}$ such that

$$
\begin{aligned}
M_{\alpha_{2}, \beta_{2}}^{v}\left(z_{1}\right) & \neq 0 \text { for }\left|z_{1}-d\right|<a_{0}, \\
\text { and }\left|M_{\alpha_{2}, \beta_{2}}^{v}\left(d \delta^{1 / \eta}\right)\right| & \approx \delta^{p_{\nu} / \eta},
\end{aligned}
$$

for all $\alpha_{2}+\beta_{2}=q_{v}$ with all $v=1, \ldots, N-1$. Property (16) means that there is $\left(\alpha^{\prime}, \beta^{\prime}\right)$ with terms mixed in $z_{2}$ and $\bar{z}_{2}$ variables for $\left|z_{1}-d\right|<a_{0}$. Let $|d|=1$ be the constant (direction) in (16) and we will fix $d$ in the rest of this paper. In the sequel, we set $\widehat{z}_{l}$ equal to $z_{l}$ or $\bar{z}_{l}, l=1,2,3$.

Remark 9. (a) $\left\{t_{k}\right\}$ defined in (12) is strictly decreasing on $k$.

(b) Each of the summation parts of (14) contains the terms of the form $\widehat{z}_{1}^{t_{k}} \widehat{z}_{2}^{k}$ where $\left(t_{k}, k\right)$ 's are the pairs, defined in (12), on the polyline $L$.

(c) Each term of the first summation part in (14) is pure in $z_{2}$ or $\bar{z}_{2}$ variables.

(d) Each term of the second summation part in (14) has terms mixed in $z_{2}$ and $\bar{z}_{2}$, and it corresponds to the pair of integers $\left(p_{v}, q_{v}\right)$, the vertices of the polyline $L$.

Lemma 10. Let $d_{0}\left(z_{1}\right):=r\left(z_{1}, 0,0\right)$ be the term containing only $z_{1}$ or $\bar{z}_{1}$ variables in the first sum of (14). Then

$$
\left|d_{0}\left(z_{1}\right)\right| \approx\left|z_{1}\right|^{\eta}
$$

Proof. From (8) and (14), we see that $\left|r\left(z_{1}, 0,0\right)\right| \leqslant\left|z_{1}\right|^{\eta}$. On the other hand, since the regular 1-type at $0 \in b \Omega$ is equal to $\eta=T_{\Omega}^{r e g}(0)$, there is $\widetilde{c}_{1}>0$ such that $\left|r\left(z_{1}, 0,0\right)\right| \geq \widetilde{c}_{1}\left|z_{1}\right|^{\eta}$.

In the sequel, we let $V$ be a small neighborhood of $z_{0}=0 \in b \Omega$ where $r(z)$ has expression as in (14). Since $\left(\partial r / \partial z_{3}\right)(0) \neq 0$, we may assume that $\left|\left(\partial r / \partial z_{3}\right)(z)\right| \geq c_{0}$ for all $z \in V$ for a uniform constant $c_{0}>0$ by shrinking $V$ if necessary. For each fixed $\delta>0$ and for each $z=\left(z_{1}, z_{2}, z_{3}\right) \in$ $V$ satisfying $\left|z_{1}-d \delta^{1 / \eta}\right|<\gamma \delta^{1 / \eta}$, for a sufficiently small $\gamma>0$ to be chosen, we set $\pi(z)=\left(z_{1}, 0, e_{\delta}\right):=\tilde{z} \in b \Omega$, where $\pi(z)$ 
is the composition of the projection onto $z_{1} z_{3}$ plane and then the projection onto $b \Omega$ along the $\operatorname{Re} z_{3}$ direction. Using the Taylor series method in $z_{3}$ variable about $e_{\delta}$, we see that

$$
r\left(z_{1}, 0,0\right)=2 \operatorname{Re}\left[\frac{\partial r(\widetilde{z})}{\partial z_{3}}\left(-e_{\delta}\right)\right]+\mathcal{O}\left(e_{\delta}^{2}\right) .
$$

Since $\left|e_{\delta}\right| \ll 1$ and $2 \operatorname{Re}\left(\partial r / \partial z_{3}\right)=1+\mathcal{O}(|z|) \geq 1 / 2$ on $V$, it follows from (17) that

$$
\left|e_{\delta}\right| \approx\left|z_{1}\right|^{\eta}
$$

for $z_{1}$ near 0

Now for each small $\delta>0$, set $z(\delta):=\left(d \delta^{1 / \eta}, 0,0\right)$ and set $\widetilde{z}^{\delta}=\pi(z(\delta))=\left(d \delta^{1 / \eta}, 0, e_{\delta}\right) \in b \Omega$. For a small constant $b>0$ to be chosen, set $z^{\delta}=\left(d \delta^{1 / \eta}, 0, e_{\delta}-b \delta\right) \in \Omega$, and for a fixed small $\delta_{0}>0$ satisfying $r\left(z^{\delta_{0}}\right)<0$, set

$$
\begin{aligned}
C_{b} & \left(z_{0}, \delta_{0}\right) \\
& :=\left\{z^{\delta}: z^{\delta}=\left(d \delta^{1 / \eta}, 0, e_{\delta}-b \delta\right), 0 \leq \delta \leq \delta_{0}\right\} \\
& \subset \Omega \cup\left\{z_{0}\right\},
\end{aligned}
$$

connecting $z^{\delta_{0}} \in V \cap \Omega$ and $z_{0}=0 \in b \Omega$.

Following the same arguments as in the proof of Proposition 1.2 in [2], for each fixed $\widetilde{z} \in V$, we can construct special coordinates about $\widetilde{z}$ so that, in terms of new coordinates, there is no pure terms in $z_{2}$ or $\bar{z}_{2}$ variables in the first summation part of $r(z)$ in (14). We will fix $z_{1}$ variable and consider the coordinate changes only on $z^{\prime \prime}=\left(z_{2}, z_{3}\right)$ variables.

Proposition 11. For each fixed $\widetilde{z}=\left(\widetilde{z}_{1}, \widetilde{z}_{2}, \widetilde{z}_{3}\right) \in V$, there is a holomorphic coordinate system $z^{\prime \prime}=\Phi_{\widetilde{z}}\left(\zeta^{\prime \prime}\right)=\left(\zeta_{2}, \Phi_{3}\left(\zeta^{\prime \prime}\right)\right)$ such that in the new coordinates $\zeta^{\prime \prime}$ defined by

$$
\Phi_{\widetilde{z}}\left(\zeta^{\prime \prime}\right)=\left(\widetilde{z}_{2}+\zeta_{2}, \Phi_{3}\left(\zeta^{\prime \prime}\right)\right)
$$

where

$$
\Phi_{3}\left(\zeta^{\prime \prime}\right)=\widetilde{z}_{3}+\left(\frac{\partial r}{\partial z_{3}}(\widetilde{z})\right)^{-1}\left(\frac{\zeta_{3}}{2}-\sum_{l=1}^{m} c_{l}(\widetilde{z}) \zeta_{2}^{l}\right)
$$

and where $c_{l}(\widetilde{z}), l=2,3, \ldots, m$, depends smoothly on $\widetilde{z}$, the function given by $\rho\left(\widetilde{z}_{1}, \zeta^{\prime \prime}\right):=r\left(\widetilde{z}_{1}, \Phi_{\widetilde{z}}\left(\zeta^{\prime \prime}\right)\right)$ satisfies

$$
\begin{aligned}
\rho\left(\widetilde{z}_{1}, \zeta^{\prime \prime}\right)= & r(\widetilde{z})+\operatorname{Re} \zeta_{3}+\sum_{\substack{j+k=2 \\
j, k>0}}^{m} a_{j, k}\left(\widetilde{z}_{1}\right) \zeta_{2}^{j} \bar{\zeta}_{2}^{k} \\
& +\mathcal{O}\left(\left|\zeta_{3}\right||\zeta|+\left|\zeta_{2}\right|^{m+1}\right) .
\end{aligned}
$$

Proof. For $\widetilde{z} \in V$, define

$$
\begin{aligned}
& \Phi^{1}\left(w^{\prime \prime}\right)=\left(\widetilde{z}_{2}+w_{2}, \widetilde{z}_{3}\right. \\
& \left.+\left(\frac{\partial r}{\partial z_{3}}(\widetilde{z})\right)^{-1}\left(\frac{w_{3}}{2}-\frac{\partial r}{\partial z_{2}}(\widetilde{z}) w_{2}\right)\right),
\end{aligned}
$$

where $w^{\prime \prime}=\left(w_{2}, w_{3}\right)$. Then we have

$$
\begin{aligned}
\rho_{2}\left(\widetilde{z}_{1}, w^{\prime \prime}\right) & :=r\left(\widetilde{z}_{1}, \Phi^{1}\left(w^{\prime \prime}\right)\right) \\
& =r(\widetilde{z})+\operatorname{Re} w_{3}+\mathcal{O}\left(\left|w^{\prime \prime}\right|^{2}\right) .
\end{aligned}
$$

Assume that (22) and (23) hold for $l \geq 2$. That is, we have defined $\Phi^{l-1}: \mathbb{C}^{2} \longrightarrow \mathbb{C}^{2}$ so that $\rho_{l}\left(\widetilde{z}_{1}, w^{\prime \prime}\right):=r\left(\widetilde{z}_{1}, \Phi^{l-1}\left(w^{\prime \prime}\right)\right)$ can be written as

$$
\begin{aligned}
\rho_{l}\left(\widetilde{z}_{1}, w^{\prime \prime}\right)= & \operatorname{Re} w_{3}+\sum_{\substack{j+k=2 \\
j, k>0}}^{l-1} a_{j, k}^{l-1}(\widetilde{z}) w_{2}^{j} \bar{w}_{2}^{k} \\
& +\mathcal{O}\left(\left|w_{3}\right|\left|w^{\prime \prime}\right|+\left|w_{2}\right|^{l}\right) .
\end{aligned}
$$

If we define $\Phi^{l}=\Phi^{l-1} \circ \phi^{l}$, where

$$
\phi^{l}\left(\zeta^{\prime \prime}\right)=\left(\zeta_{2}, \zeta_{3}-\frac{2}{l !} \frac{\partial^{l} \rho_{l}}{\partial w_{2}^{l}}\left(\widetilde{z}_{1}, 0,0\right) \zeta_{2}^{l}\right),
$$

then $\rho_{l+1}\left(\widetilde{z}_{1}, \zeta^{\prime \prime}\right):=r\left(\widetilde{z}_{1}, \Phi^{l}\left(\zeta^{\prime \prime}\right)\right)$ satisfies (26) for $l$ replaced by $l+1$. If we proceed up to $l=m$ and set $\Phi_{\widetilde{z}}=\Phi^{m}=$ $\Phi^{1} \circ \phi^{2} \circ \cdots \circ \phi^{m}$, then by setting $\rho=\rho_{m+1}=r\left(\widetilde{z}, \Phi_{\widetilde{z}}(\cdot)\right)$, we see that (22) and (23) hold.

In the sequel, we will use the coordinate changes in Proposition 11 only at $\widetilde{z}=\left(\widetilde{z}_{1}, 0, \widetilde{z}_{3}\right) \in V$, (in particular at $\widetilde{z}^{\delta}=\left(d \delta^{1 / \eta}, 0, e_{\delta}\right) \in b \Omega$ in Section 3$)$. We want to study the dependence of $\Phi_{\widetilde{z}}$ about $\widetilde{z}$. For each $\widetilde{z}=\left(\widetilde{z}_{1}, 0, \widetilde{z}_{3}\right) \in V$, set $c_{0}(\widetilde{z}):=\left(\partial r / \partial z_{3}\right)(\widetilde{z})=1+\mathcal{O}(|\widetilde{z}|)$, and we note that

$$
c_{l}(\widetilde{z})=\frac{1}{l !} \frac{\partial^{l} \rho_{l}}{\partial w_{2}^{l}}\left(\widetilde{z}_{1}, 0,0\right), \quad l=1,2, \ldots, m,
$$

where $\rho_{l}$ is defined in the inductive step of the proof of Proposition 11. Set

$$
\begin{aligned}
& e_{0}(\widetilde{z})=\frac{1}{2} c_{0}(\widetilde{z})^{-1} \\
& e_{1}(\widetilde{z})=-c_{0}(\widetilde{z})^{-1} \frac{\partial r}{\partial z_{2}}(\widetilde{z}) \text { and } \\
& e_{l}(\widetilde{z})=-c_{0}(\widetilde{z})^{-1} c_{l}(\widetilde{z}) \quad l=2, \ldots, m
\end{aligned}
$$

and set $\rho_{0}=r$. Then $\rho_{1}\left(\widetilde{z}_{1}, \zeta^{\prime \prime}\right)=r\left(\widetilde{z}_{1}, \zeta_{2}, \widetilde{z}_{3}+e_{0} \zeta_{3}\right)$ and

$$
\rho_{i+1}=\rho_{i}\left(\widetilde{z}_{1}, \zeta_{2}, \zeta_{3}+e_{i}(\widetilde{z}) \zeta_{2}^{i}\right), \quad i=1,2, \ldots, m
$$

To study the dependence of $\Phi_{\widetilde{z}}$ and hence dependence of $a_{j, k}\left(\widetilde{z}_{1}\right)$ about $\widetilde{z}_{1}$ in (23), we thus need to study the dependence of $e_{l}(\widetilde{z})$ on $\widetilde{z}$ variable. For a convenience, set $\widetilde{z}=\left(z_{1}, 0, z_{3}\right)$, i.e., remove tilde's, and assume that $\widetilde{z}$ satisfies

$$
\begin{array}{r}
\left|z_{1}-d \delta^{1 / \eta}\right|<\gamma \delta^{1 / \eta}, \\
\text { and }\left|z_{3}\right| \lesssim\left|z_{1}\right|^{\eta},
\end{array}
$$


for a sufficiently small $\gamma>0$ to be chosen. In view of (19), we see that $\widetilde{z}^{\delta}=\left(d \delta^{1 / \eta}, 0, e_{\delta}\right)$ satisfies (31). In following we let $z$ be the given coordinates, and we let $\zeta$ be the coordinates obtained from holomorphic coordinates changes of $z$, as in l-th step of coordinate changes in the proof of Proposition 11. Also we let $D_{k}^{s}$ (resp., $\widetilde{D}_{k}^{s}$ ), $k=1,2,3$, denote any partial derivative operator of order $s$ with respect to $z_{k}$ and $\bar{z}_{k}$ (resp., $\zeta_{k}$ and $\bar{\zeta}_{k}$ ) variables. According to the coordinate changes in Proposition 11, we note that $D_{1}=\widetilde{D}_{1}$.

Proposition 12. Assume that $\widetilde{z}=\left(z_{1}, 0, z_{3}\right) \in V$ satisfies (31). Then for each $i=0,1, \ldots, m+1$, we have

$$
\left|D_{1}^{l_{1}} \widetilde{D}_{2}^{k} \rho_{i}(\widetilde{z})\right| \lesssim\left|z_{1}\right|^{t_{k}-l_{1}}, \quad 0 \leq k \leq m, 0 \leq l_{1} \leq \eta,
$$

and for each $\alpha_{2}, \beta_{2}>0$ with $\alpha_{2}+\beta_{2}=q_{\nu}$, for some $q_{\nu}$ in (14), we have

$$
\begin{aligned}
\left|D_{1}^{l_{1}} \widetilde{D}_{2}^{q_{v}} \rho_{i}(\widetilde{z})\right| \approx\left|D_{1}^{l_{1}} D_{2}^{q_{v}} r(\widetilde{z})\right| & \approx\left|z_{1}\right|^{p_{v}-l_{1}}, \\
& l_{1} \leq p_{\nu}, 2 \leq i \leq m+1 .
\end{aligned}
$$

Proof. We will prove by induction on $i$. From (14), (17), and (31) one obtains

$$
\begin{aligned}
\left|D_{1}^{l_{1}} D_{2}^{k} r(\widetilde{z})\right| & \lesssim\left|z_{1}\right|^{\eta-l_{1}}+\left|z_{1}\right|^{t_{k}-l_{1}}+\left|z_{3}\right|+\left|z_{1}\right|^{\left[t_{k}\right]+1-l_{1}} \\
& \leq\left|z_{1}\right|^{t_{k}-l_{1}}
\end{aligned}
$$

and hence (32) follows for $i=0$. Since $\rho_{1}\left(z_{1}, \zeta^{\prime \prime}\right)=$ $r\left(z_{1}, \zeta_{2}, z_{3}+e_{0} \zeta_{3}\right)$, it follows, from (31) and chain rule, that

$$
\left|D_{1}^{l_{1}} \widetilde{D}_{2}^{k} \rho_{1}(\widetilde{z})\right| \lesssim\left|D_{1}^{l_{1}} \widetilde{D}_{2}^{k} r(\widetilde{z})\right|+\left|z_{3}\right| \lesssim\left|z_{1}\right|^{t_{k}-l_{1}},
$$

because we are evaluating at $\widetilde{\zeta}=\left(z_{1}, 0,0\right)$. This proves (32) for $i=1$.

By induction, assume that (32) holds for $i=0,1, \ldots, s$. For the $e_{i}(\widetilde{z})$ defined in (29), it follows, from (34) and induction hypothesis, that

$$
\left|D_{1}^{l_{1}} e_{i}(\widetilde{z})\right| \lesssim \sum_{j=0}^{l_{1}}\left|D_{1}^{j} D_{2}^{i} \rho_{i}(\widetilde{z})\right| \lesssim \sum_{j=0}^{l_{1}}\left|z_{1}\right|^{t_{i}-j} \lesssim\left|z_{1}\right|^{t_{i}-l_{1}}
$$

for $i=1, \ldots, s$. Since we are evaluating at $\zeta_{2}=0$, it follows, for $s \geq 1$, that

$$
\begin{aligned}
D_{1}^{l_{1}} \widetilde{D}_{2}^{k} \rho_{s+1}(\widetilde{z}) & =D_{1}^{l_{1}} \widetilde{D}_{2}^{k} \rho_{s}(\widetilde{z}), \quad \text { if } k<s \text {, and } \\
& =D_{1}^{l_{1}} \widetilde{D}_{2}^{k} \rho_{s}(\widetilde{z})+\mathcal{O}\left(\sum_{j=0}^{l_{1}} D_{1}^{j} e_{s}(\widetilde{z})\right),
\end{aligned}
$$

if $k \geq s$.

By (30), (36), and (37) and by induction, (32) holds for $i=s+1$ because $t_{k} \leq t_{s}$ if $k \geq s$.

Now we prove (33). Assume $\alpha_{2}+\beta_{2}=q_{v}$ with $\alpha_{2}>0$, $\beta_{2}>0$ where $\left(p_{v}, q_{\nu}\right)$ are the pairs corresponding to the second summation part of (14). Note that the first summation part of (14) will be annihilated by $D_{2}^{q_{v}}$ because it contains the pure terms of $z_{2}$ or $\bar{z}_{2}$ mixed with $\bar{z}_{1}^{k}$. Thus it follows from (14), (16), and (31) that

$$
\begin{aligned}
\left|D_{1}^{l_{1}} D_{2}^{q_{v}} r(\widetilde{z})\right| \approx & \left|D_{1}^{l_{1}} M_{\alpha_{2}, \beta_{2}}^{v}\left(z_{1}\right)\right| \\
& +\mathcal{O}\left(\left|z_{3}\right|+\left|z_{1}\right|^{p_{\nu}+1-l_{1}}\right) \approx\left|z_{1}\right|^{p_{v}-l_{1}} .
\end{aligned}
$$

Since $\rho_{1}\left(z_{1}, \zeta^{\prime \prime}\right)=r\left(z_{1}, \zeta_{2}, z_{3}+e_{0} \zeta_{3}\right)$, it follows from (31) and (38) that

$$
\left|D_{1}^{l_{1}} \widetilde{D}_{2}^{q_{v}} \rho_{1}(\widetilde{z})\right| \approx\left|D_{1}^{l_{1}} \widetilde{D}_{2}^{q_{v}} r(\widetilde{z})\right|+\mathcal{O}\left(\left|z_{3}\right|\right) \approx\left|z_{1}\right|^{p_{v}-l_{1}} .
$$

Similarly, since $\rho_{2}\left(z_{1}, \zeta^{\prime \prime}\right)=\rho_{1}\left(z_{1}, \zeta_{2}, \zeta_{3}+e_{1} \zeta_{2}\right)$, it follows from (36) that

$$
\begin{aligned}
\left|D_{1}^{l_{1}} \widetilde{D}_{2}^{q_{v}} \rho_{2}(\widetilde{z})\right| & \approx\left|D_{1}^{l_{1}} \widetilde{D}_{2}^{q_{v}} \rho_{1}(\widetilde{z})\right|+\sum_{j=0}^{l_{1}}\left|D_{1}^{j} e_{1}\right| \\
& \approx\left|z_{1}\right|^{p_{v}-l_{1}},
\end{aligned}
$$

because $p_{v}=t_{q_{v}}<t_{1}$ for $q_{v} \geq 2$. This proves (33) for $i=2$.

By induction assume that (33) holds for $i=2, \ldots, s$. If $k=q_{v}=\alpha_{v}+\beta_{v}$ with $\alpha_{v}>0$ and $\beta_{v}>0$, that is, if $\widetilde{D}_{2}^{k}$ has mixed derivatives of $\partial / \partial \zeta_{2}$ and $\partial / \partial \bar{\zeta}_{2}$, we note that (37) becomes

$$
\begin{aligned}
D_{1}^{l_{1}} \widetilde{D}_{2}^{k} \rho_{s+1}(\widetilde{z}) & =D_{1}^{l_{1}} \widetilde{D}_{2}^{k} \rho_{s}(\widetilde{z}), \quad \text { if } k \leq s, \text { and } \\
& =D_{1}^{l_{1}} \widetilde{D}_{2}^{k} \rho_{s}(\widetilde{z})+\mathcal{O}\left(\sum_{j=0}^{l_{1}} D_{1}^{j} e_{s}(\widetilde{z})\right),
\end{aligned}
$$

if $k>s$.

If $k=q_{v} \leq s$, (33) follows from (41) and induction hypothesis of (33). If $q_{v}>s$, it follows, from (36), (41), and induction hypothesis of (33), that

$$
\begin{aligned}
\left|D_{1}^{l_{1}} \widetilde{D}_{2}^{q_{v}} \rho_{s+1}(\widetilde{z})\right|= & \left|D_{1}^{l_{1}} \widetilde{D}_{2}^{q_{v}} \rho_{s}(\widetilde{z})\right| \\
& +\mathcal{O}\left(\sum_{j=0}^{l_{1}}\left|D_{1}^{j} e_{s}(\widetilde{z})\right|\right) \approx\left|z_{1}\right|^{p_{v}-l_{1}},
\end{aligned}
$$

because $t_{s}>p_{v}=t_{q_{v}}$ for $q_{v}>s$. Therefore (33) is proved for $i=s+1$.

Recall the expression of $\rho=\rho_{m+1}$ and coefficient functions $a_{j, k}\left(\widetilde{z}_{1}\right)$ in $(23)$.

Corollary 13. Assume that $\widetilde{z}=\left(z_{1}, 0, z_{3}\right)$ satisfies (31). Then

$$
\left|D_{1}^{l_{1}} a_{j, k}\left(z_{1}\right)\right| \lesssim\left|z_{1}\right|^{t_{j+k}-l_{1}}
$$

and if $j+k=q_{\nu}$ for some $q_{\nu}$ in (14), then

$$
\left|D_{1}^{l_{1}} a_{j, k}\left(z_{1}\right)\right| \approx\left|z_{1}\right|^{p_{v}-l_{1}} .
$$


Proof. From (23) we see that

$$
D_{1}^{l_{1}} a_{j, k}\left(z_{1}\right)=D_{1}^{l_{1}} \widetilde{D}_{2}^{j+k} \rho(\widetilde{\zeta}),
$$

where $\widetilde{\zeta}=\left(z_{1}, 0,0\right)$ and $j, k>0$. Hence it follows from (32) that

$$
\left|D_{1}^{l_{1}} a_{j, k}\left(z_{1}\right)\right|=\left|D_{1}^{l_{1}} \widetilde{D}_{2}^{j+k} \rho(\widetilde{\zeta})\right| \lesssim\left|z_{1}\right|^{t_{j+k}-l_{1}} .
$$

Assume $q_{v}=j+k \leq m$ for some $q_{v}$. Thus $j, k>0$ and it follows from (23), (33), and (41) that

$$
\begin{aligned}
\left|D_{1}^{l_{1}} a_{j, k}\left(z_{1}\right)\right| & =\left|D_{1}^{l_{1}} \widetilde{D}_{2}^{q_{v}} \rho(\widetilde{\zeta})\right|=\left|D_{1}^{l_{1}} \widetilde{D}_{2}^{q_{v}} \rho_{q_{v}}(\widetilde{\zeta})\right| \\
& \approx\left|z_{1}\right|^{t_{q_{v}}-l_{1}}=\left|z_{1}\right|^{p_{v}-l_{1}},
\end{aligned}
$$

because $t_{q_{v}}=p_{\nu}$.

Remark 14. Suppose that $q_{\nu-1}<l \leq q_{\nu}$ and $p_{\nu} \leq t_{l}<p_{\nu-1}$. Then $\left(p_{v}, q_{\nu}\right),\left(t_{l}, l\right)$, and $\left(p_{\nu-1}, q_{\nu-1}\right)$ are colinear points. From the standard interpolation method, we have

$$
a^{t_{l}} b^{l} \leq a^{p_{\nu}} b^{q_{\nu}}+a^{p_{\nu-1}} b^{q_{\nu-1}},
$$

for all sufficiently small $a, b \geq 0$. Assume that $j, k>0$ and $l=j+k \neq q_{v}$ for any of $v=1,2, \ldots, N$. Therefore it follows from (43) and (48) that

$$
\begin{aligned}
\left|a_{j, k}\left(z_{1}\right) \zeta_{2}^{j} \bar{\zeta}_{2}^{k}\right| & \lesssim\left|z_{1}\right|^{t_{j+k}}\left|\zeta_{2}\right|^{j+k} \\
& \leq\left|z_{1}\right|^{p_{\nu}}\left|\zeta_{2}\right|^{q_{\nu}}+\left|z_{1}\right|^{p_{\nu-1}}\left|\zeta_{2}\right|^{q_{\nu-1}} .
\end{aligned}
$$

Therefore the terms of the form $a_{j, k}\left(z_{1}\right) \zeta_{2}^{j} \bar{\zeta}_{2}^{k}$, with $j+k=q_{\nu}$ for some $q_{v}$, in the summation part in (23), are the major terms which bounds the other summation terms from above.

In the sequel, we assume that $\widetilde{z}=\left(z_{1}, 0, z_{3}\right)$ satisfies (31). As in Section 1 in [2], for each $\widetilde{z}=\left(z_{1}, 0, z_{3}\right)$, set

$$
\begin{aligned}
A_{l}(\widetilde{z})=A_{l}\left(z_{1}\right)=\max \left\{\left|a_{j, k}\left(z_{1}\right)\right| ; j+k\right. & =l\}, \\
& l=2, \ldots, m .
\end{aligned}
$$

In view of Remark 14, we will consider $A_{l}\left(z_{1}\right)$ only for $l=q_{v}$, $0 \leq v \leq N-1$. From (9) and (44) we note that

$$
\begin{aligned}
& \left|A_{q_{0}}\left(z_{1}\right)\right|=\left|A_{m}\left(z_{1}\right)\right| \approx 1 \text {, and } \\
& \left|A_{q_{v}}\left(z_{1}\right)\right| \approx\left|z_{1}\right|^{p_{v}}, \quad 1 \leq \nu \leq N-1,
\end{aligned}
$$

because $q_{0}=m$. For each sufficiently small $\delta>0$, set

$$
\begin{aligned}
\tau(\widetilde{z}, \delta) & =\tau\left(z_{1}, \delta\right) \\
& =\min \left\{\left(\frac{\delta}{A_{q_{v}}\left(z_{1}\right)}\right)^{1 / q_{v}} ; 0 \leq \nu \leq N-1\right\},
\end{aligned}
$$

and set

$$
\begin{aligned}
T(\tilde{z}, \delta) & =T\left(z_{1}, \delta\right) \\
& =\min \left\{q_{\nu} ;\left(\frac{\delta}{A_{q_{\nu}}\left(z_{1}\right)}\right)^{1 / q_{\nu}}=\tau\left(z_{1}, \delta\right)\right\} .
\end{aligned}
$$

From (51) and (52), we see that if $\delta^{\prime}<\delta$, then

$$
\left(\frac{\delta^{\prime}}{\delta}\right)^{1 / 2} \tau(\widetilde{z}, \delta) \leq \tau\left(\widetilde{z}, \delta^{\prime}\right) \leq\left(\frac{\delta^{\prime}}{\delta}\right)^{1 / m} \tau(\widetilde{z}, \delta)
$$

Lemma 15. For each $0<\epsilon \leq 1, T(\widetilde{z}, \epsilon \delta) \leq T(\widetilde{z}, \delta)$.

Proof. Set $q_{v}^{\epsilon}=T(\widetilde{z}, \epsilon \delta)$ and $q_{v}=T(\widetilde{z}, \delta)$. Then

$$
\begin{aligned}
\tau(\widetilde{z}, \epsilon \delta) & =\left(\frac{\epsilon \delta}{A_{q_{\nu}^{e}}(\widetilde{z})}\right)^{1 / q_{\nu}^{\epsilon}}=\epsilon^{1 / q_{\nu}^{\epsilon}}\left(\frac{\delta}{A_{q_{\nu}^{e}}(\widetilde{z})}\right)^{1 / q_{\nu}^{e}} \\
& \geq \epsilon^{1 / q_{\nu}^{\epsilon}} \tau(\widetilde{z}, \delta)=\epsilon^{1 / q_{\nu}^{\epsilon}}\left(\frac{\delta}{A_{q_{\nu}}(\widetilde{z})}\right)^{1 / q_{\nu}} \\
& =\epsilon^{1 / q_{\nu}^{\epsilon}-1 / q_{\nu}}\left(\frac{\epsilon \delta}{A_{q_{\nu}}(\widetilde{z})}\right)^{1 / q_{\nu}} \\
& \geq \epsilon^{1 / q_{\nu}^{\epsilon}-1 / q_{\nu}} \tau(\widetilde{z}, \epsilon \delta) .
\end{aligned}
$$

Therefore $q_{\nu}^{\epsilon} \leq q_{\nu}$ because $0<\epsilon \leq 1$.

Proposition 16. Assume $\widetilde{z}=\left(z_{1}, 0, z_{3}\right)$ satisfies (31). Then

$$
\tau(\widetilde{z}, \delta) \approx \tau\left(\widetilde{z}^{\delta}, \delta\right)
$$

where $\widetilde{z}^{\delta}=\left(d \delta^{1 / \eta}, 0, e_{\delta}\right)$.

Proof. By (31), we note that $\left|z_{1}\right| \approx \delta^{1 / \eta}$. Assume that $T\left(\widetilde{z}^{\delta}\right.$, $\delta)=q_{v}$. Then $A_{q_{v}}\left(\widetilde{z}^{\delta}\right) \approx \delta^{p_{v} / \eta}$ by (51). Therefore it follows, from (50) and (52), that

$$
A_{q_{\nu}}(\widetilde{z}) \approx\left|z_{1}\right|^{p_{\nu}} \approx \delta^{p_{\nu} / \eta} \approx A_{q_{\nu}}\left(\widetilde{z}^{\delta}\right)
$$

and hence it follows from (52) and (53) that

$$
\tau\left(\widetilde{z}^{\delta}, \delta\right)^{q_{\nu}}=\frac{\delta}{A_{q_{\nu}}\left(\widetilde{z}^{\delta}\right)} \approx \frac{\delta}{A_{q_{\nu}}(\widetilde{z})} \geq \tau(\widetilde{z}, \delta)^{q_{\nu}} .
$$

Thus $\tau\left(\widetilde{z}^{\delta}, \delta\right) \gtrsim \tau(\widetilde{z}, \delta)$ follows. Similarly, one can show that $\tau\left(\widetilde{z}^{\delta}, \delta\right) \lesssim \tau(\widetilde{z}, \delta)$.

Let $0<\sigma<1$ be a small constant to be determined (in Remark 22). By Lemma 15, $T\left(\widetilde{z}^{\delta}, \sigma \delta\right) \leq T\left(\widetilde{z}^{\delta}, \delta\right)$ for each $0<$ $\sigma<1$, independent of $\delta>0$. Therefore there is a smallest integer $s=s\left(\widetilde{z}^{\delta}\right), 0 \leq s \leq m-1$, such that

$$
T\left(\widetilde{z}^{\delta}, \sigma^{s+1} \delta\right)=T\left(\widetilde{z}^{\delta}, \sigma^{s} \delta\right):=t_{s} .
$$

Then $t_{s}=q_{\nu(s)}$ for some $q_{\nu(s)}$ by (53). In following, for the fixed integer $s=s\left(\widetilde{z}^{\delta}\right)$ in (59), set $\delta_{s}=\sigma^{s} \delta, \tau_{s}:=\tau\left(\widetilde{z}^{\delta}, \delta_{s}\right)$, and $\tau_{1}=\delta^{1 / \eta}$ as usual. If we define $\Phi_{\widetilde{z}}(\zeta)=\left(\zeta_{1}, \zeta_{2}, \Phi_{3}\left(\zeta^{\prime \prime}\right)\right)$, where $\Phi_{3}\left(\zeta^{\prime \prime}\right)$ is defined in (22), we may regard that $\Phi_{\widetilde{z}}(\zeta): \mathbb{C}^{3} \longrightarrow$ 
$\mathbb{C}^{3}$ is a biholomorphism. For each $\widetilde{z}^{\delta}=\left(d \delta^{1 / \eta}, 0, e_{\delta}\right) \in b \Omega$, set $\widetilde{\zeta}^{\delta}=\left(d \delta^{1 / \eta}, 0,0\right)=\Phi_{\widetilde{z}^{\delta}}^{-1}\left(\widetilde{z}^{\delta}\right)$. For each small $\gamma>0$, define

$$
\begin{aligned}
& R_{\gamma \delta}^{s}\left(\widetilde{\zeta}^{\delta}\right) \\
& \quad:=\left\{\zeta\left|\zeta_{1}-d \delta^{1 / \eta}\right|<\gamma \tau_{1},\left|\zeta_{2}\right|<\gamma \tau_{s},\left|\zeta_{3}\right|<\gamma \delta_{s}\right\},
\end{aligned}
$$

and,

$$
Q_{\gamma \delta}^{s}\left(\widetilde{z}^{\delta}\right):=\left\{\Phi_{\widetilde{z}^{\delta}}(\zeta) ; \zeta \in R_{\gamma \delta}^{s}\left(\widetilde{\zeta}^{\delta}\right)\right\},
$$

and set $R_{\gamma \delta}^{0}\left(\widetilde{\zeta}^{\delta}\right)=R_{\gamma \delta}\left(\widetilde{\zeta}^{\delta}\right)$ and $Q_{\gamma \delta}^{0}\left(\widetilde{z}^{\delta}\right)=Q_{\gamma \delta}\left(\widetilde{z}^{\delta}\right)$ when $s=0$.

Proposition 17. The function $\rho=r \circ \Phi_{\bar{z}^{\delta}}$ satisfies

$$
\begin{aligned}
\left|D_{1}^{l_{1}} \rho\left(\widetilde{\zeta}^{\delta}\right)\right| & \leqslant \delta \tau_{1}^{-l_{1}}, \text { and, } \\
\left|D_{1}^{l_{1}} \widetilde{D}_{2}^{k} \rho\left(\widetilde{\zeta}^{\delta}\right)\right| & \leqslant \delta_{s} \tau_{1}^{-l_{1}} \tau_{s}^{-k}, \quad 1 \leq k \leq m .
\end{aligned}
$$

Proof. Recall that $\rho=\rho_{m+1}$, and $\left|z_{1}\right|=\delta^{1 / \eta}$ in (32). When $k=0$, it follows from (12) $\left(t_{0}=\eta\right)$ and (32) that

$$
\left|D^{l_{1}} \rho\left(\widetilde{\zeta}^{\delta}\right)\right| \lesssim\left(\delta^{1 / \eta}\right)^{\eta-l_{1}}=\delta \tau_{1}^{-l_{1}} .
$$

Assume $1 \leq k \leq m$. Then by (12), $q_{v-1}<k \leq q_{\nu}$ for some $v$, and hence it follows that $p_{v} \leq t_{k} \leq p_{v-1}$. Therefore one obtains, from (48)-(52), that

$$
\begin{aligned}
\left|z_{1}\right|^{t_{k}} \tau_{s}^{k} & \leqslant\left|z_{1}\right|^{p_{\nu-1}} \tau_{s}^{q_{\nu-1}}+\left|z_{1}\right|^{p_{\nu}} \tau_{s}^{q_{\nu}} \\
& \leqslant A_{q_{\nu-1}} \tau_{s}^{q_{\nu-1}}+A_{q_{\nu}} \tau_{s}^{q_{\nu}} \leq \delta_{s} .
\end{aligned}
$$

From (32) and (63), it follows that

$$
\begin{aligned}
\left|D_{1}^{l_{1}} \widetilde{D}_{2}^{k} \rho\left(\widetilde{\zeta}^{\delta}\right)\right| & \leq\left|z_{1}\right|^{t_{k}-l_{1}}=\left(\left|z_{1}\right|^{t_{k}} \tau_{s}^{k}\right)\left|z_{1}\right|^{-l_{1}} \tau_{s}^{-k} \\
& \leqslant \delta_{s} \tau_{1}^{-l_{1}} \tau_{s}^{-k} .
\end{aligned}
$$

Using the $z$ coordinates defined in (14), set

$$
\begin{aligned}
& L_{3}=\frac{\partial}{\partial z_{3}} \text { and } \\
& L_{k}=\frac{\partial}{\partial z_{k}}-\left(\frac{\partial r}{\partial z_{3}}\right)^{-1} \frac{\partial r}{\partial z_{k}} \frac{\partial}{\partial z_{3}}:=\frac{\partial}{\partial z_{k}}+b_{k}(z) \frac{\partial}{\partial z_{3}}, \\
& k=1,2 .
\end{aligned}
$$

Then $L_{k}, k=1,2$, are tangential holomorphic vector fields and $\left|L_{3} r\right| \geq c_{0}>0$ on $V \cap \Omega$ for a uniform constant $c_{0}>0$. For any $j, k$ with $j, k>0$, define

$$
\mathscr{L}_{j, k} \partial \bar{\partial} r(z)=\underbrace{L_{2} \ldots L_{2}}_{(j-1) \text { times }} \underbrace{\bar{L}_{2} \ldots \bar{L}_{2}}_{(k-1) \text { times }} \partial \bar{\partial} r\left(L_{2}, \bar{L}_{2}\right)(z) .
$$

In $\zeta$-coordinates defined by $z=\left(z_{1}, \Phi_{\widetilde{z}}\left(\zeta^{\prime \prime}\right)\right):=\Phi_{\widetilde{z}}(\zeta)$, set $L_{k}^{\prime}=\left(d \Phi_{\widetilde{z}}^{-1}\right) L_{k}, k=1,2,3$ and set $\widetilde{b}_{k}(\zeta)=b_{k}\left(\Phi_{\widetilde{z}}(\zeta)\right), k=1,2$. If we define

$$
\mathscr{L}_{j, k}^{\prime} \partial \bar{\partial} \rho(\zeta)=\underbrace{L_{2}^{\prime} \ldots L_{2}^{\prime}}_{(j-1) \text { times }} \underbrace{\bar{L}_{2}^{\prime} \ldots \bar{L}_{2}^{\prime}}_{(k-1) \text { times }} \partial \bar{\partial} \rho\left(L_{2}^{\prime}, \bar{L}_{2}^{\prime}\right)(\zeta),
$$

then by functoriality,

$$
\mathscr{L}_{j, k} \partial \bar{\partial} r(z)=\mathscr{L}_{j, k}^{\prime} \partial \bar{\partial} \rho(\zeta) .
$$

Lemma 18. There is a small constant $c_{2}>0$ such that

$$
\partial \bar{\partial} r(z)\left(L_{1}, \bar{L}_{1}\right) \geq c_{2} \delta \tau_{1}^{-2}, \quad z \in Q_{\gamma \delta}\left(\widetilde{z}^{\delta}\right),
$$

provided $\gamma>0$ is sufficiently small.

Proof. Since the level sets of $\rho$ are pseudoconvex, it follows from (61) that

$$
\begin{aligned}
\partial \bar{\partial} \rho(\zeta)\left(L_{1}^{\prime}, \bar{L}_{1}^{\prime}\right) & =\left|\frac{\partial^{2} \rho}{\partial \zeta_{1} \partial \bar{\zeta}_{1}}(\zeta)+\mathcal{O}\left(\widetilde{b}_{1}\right)\right| \\
& \geq\left|\frac{\partial^{2} \rho}{\partial \zeta_{1} \partial \bar{\zeta}_{1}}(\zeta)\right|-\widetilde{C}_{1} \delta \tau_{1}^{-1} .
\end{aligned}
$$

Recall that $d_{0}\left(z_{1}\right)=\sum_{\alpha_{1}+\beta_{1}=\eta} a_{\alpha_{1}, \beta_{1}} z_{1}^{\alpha_{1}} \bar{z}_{1}^{\beta_{1}}$ is the term which contains only $z_{1}$ or $\bar{z}_{1}$ variables in the first summation part of (14). Therefore it follows, from (17), (19), and (23), that

$$
\begin{aligned}
\left|\frac{\partial^{2} \rho}{\partial \zeta_{1} \partial \bar{\zeta}_{1}}\left(\widetilde{\zeta}^{\delta}\right)\right| & =\left|\frac{\partial^{2} r\left(\widetilde{z}^{\delta}\right)}{\partial z_{1} \partial \bar{z}_{1}}\right| \\
& =\left|\frac{\partial^{2} d_{0}\left(z_{1}\right)}{\partial z_{1} \partial \bar{z}_{1}}\right|+\mathcal{O}\left(\left|e_{\delta}\right|+\left|z_{1}\right|^{\eta-1}\right) \\
& \approx\left|z_{1}\right|^{\eta-2}=\delta \tau_{1}^{-2},
\end{aligned}
$$

because $\left|z_{1}\right|=\left|d \delta^{1 / \eta}\right|=\tau_{1}=\delta^{1 / \eta}$. If $\zeta \in R_{\gamma \delta}\left(\widetilde{\zeta}^{\delta}\right)$, it follows from (61) and (71) and by using the Taylor series method that

$$
\begin{array}{r}
\left|\frac{\partial^{2} \rho}{\partial \zeta_{1} \partial \bar{\zeta}_{1}}(\zeta)\right| \geq\left|\frac{\partial^{2} \rho}{\partial \zeta_{1} \partial \bar{\zeta}_{1}}\left(\widetilde{\zeta}^{\delta}\right)\right|-\gamma \widetilde{C}_{1} \delta \tau_{1}^{-2} \\
\geq 2 c_{2} \delta \tau_{1}^{-2}, \\
\zeta \in R_{\gamma \delta}\left(\widetilde{\zeta}^{\delta}\right),
\end{array}
$$

provided $\gamma>0$ is sufficiently small. Thus (69) follows from (68), (70), and (72).

In the sequel, we let $c_{2}$ and $C_{2}$ be the constants which may different from time to time but depend only on the derivatives of $r$ or $\rho$ up to order $\eta$. Recall that $\widetilde{b}_{k}(\zeta)=b_{k}\left(\Phi_{\widetilde{z}}(\zeta)\right), k=1,2$. By using (61), and by using Taylor series method, one obtains that

$$
\left|D_{1}^{l_{1}} D_{2}^{l_{2}} \widetilde{b}_{k}(\zeta)\right| \leq C_{2} \delta_{s} \tau_{1}^{-l_{1}} \tau_{s}^{-l_{2}} \widetilde{\tau}_{k}^{-1}, \quad \zeta \in R_{\gamma \delta}^{s}\left(\widetilde{\zeta}^{\delta}\right)
$$

provided $\gamma>0$ is sufficiently small, where $\widetilde{\tau}_{1}=\tau_{1}$ and $\widetilde{\tau}_{2}=\tau_{s}$. Note that we can write

$$
\partial \bar{\partial} \rho(\zeta)\left(L_{2}^{\prime}, \bar{L}_{2}^{\prime}\right)=\frac{\partial^{2} \rho}{\partial \zeta_{2} \partial \bar{\zeta}_{2}}+R_{1}
$$

where $R_{1}=\mathcal{O}\left(\widetilde{b}_{2}\right)$. By applying $L_{2}^{\prime}$ or $\bar{L}_{2}^{\prime}$ successively to $\partial \bar{\partial} \rho(\zeta)\left(L_{2}^{\prime}, \bar{L}_{2}^{\prime}\right)$, we obtain that

$$
D_{1}^{l_{1}} \widetilde{D}_{2}^{l_{2}} \mathscr{L}_{j, k}^{\prime} \partial \bar{\partial} \rho(\zeta)=D_{1}^{l_{1}} \widetilde{D}_{2}^{l_{2}} \frac{\partial^{j+k} \rho}{\partial \zeta_{2}^{j} \partial \bar{\zeta}_{2}^{k}}+D_{1}^{l_{1}} \widetilde{D}_{2}^{l_{2}} R_{j+k-1},
$$


where, by (73) and by using induction method, $R_{j+k-1}$ satisfies

$$
\begin{aligned}
\left|D_{1}^{l_{1}} \widetilde{D}_{2}^{l_{2}} R_{j+k-1}(\zeta)\right| \leq C_{2} \delta_{s} \tau_{1}^{-l_{1}} \tau_{s}^{-l_{2}-j-k+1}, & \\
& \zeta \in R_{\gamma \delta}^{s}\left(\widetilde{\zeta}^{\delta}\right) .
\end{aligned}
$$

Combining the estimate in (61), (75), and (76), one obtains that

$$
\left|\mathscr{L}_{j, k}^{\prime} \partial \bar{\partial} \rho(\zeta)\right| \leq C_{2} \delta_{s} \tau_{s}^{-j-k}, \quad \zeta \in R_{\gamma \delta}^{s}\left(\widetilde{\zeta}^{\delta}\right)
$$

Assume that (59) holds. Thus $t_{s}=q_{\gamma(s)}$ for some $q_{\nu(s)}$, and hence it follows from (53) that $A_{q_{\nu(s)}}\left(z_{1}\right)=\delta_{s} \tau_{s}^{-q_{\nu(s)}}$. Therefore it follows from (23) and (50) that there exist integers $j, k>0$ with $j+k=t_{s}=q_{v(s)}$, such that

$$
\begin{aligned}
\left|\frac{\partial^{j+k} \rho}{\partial \zeta_{2}^{j} \partial \bar{\zeta}_{2}^{k}}\left(\widetilde{\zeta}^{\delta}\right)\right| & =\left|a_{j, k}\left(\widetilde{z}^{\delta}\right)\right|=A_{q_{\gamma(s)}}\left(z_{1}\right)=\delta_{s} \tau_{s}^{-q_{\nu(s)}} \\
& =\delta_{s} \tau_{s}^{-j-k} .
\end{aligned}
$$

For these $j, k>0$, it follows from (61), (75), (76), and (78) and by using the Taylor series method that there are constants $c_{2}, C_{2}>0$ such that

$$
\begin{aligned}
c_{2} \delta_{s} \tau_{s}^{-j-k} \leq\left|\mathscr{L}_{j, k} \partial \bar{\partial} r(z)\right| \leq C_{2} \delta_{s} \tau_{s}^{-j-k}, & \\
& z \in Q_{\gamma \delta}^{s}\left(\widetilde{z}^{\delta}\right),
\end{aligned}
$$

provided $\gamma>0$ is sufficiently small.

Lemma 19. There is $C_{2}>0$ such that

$$
\left|\partial \bar{\partial} r(z)\left(L_{1}, \bar{L}_{2}\right)\right| \leq C_{2} \gamma \delta_{s} \tau_{1}^{-1} \tau_{s}^{-1}, \quad z \in Q_{\gamma \delta}^{s}\left(\widetilde{z}^{\delta}\right) .
$$

Proof. By functoriality, we have

$$
\begin{aligned}
\partial \bar{\partial} r(z)\left(L_{1}, \bar{L}_{2}\right) & =\partial \bar{\partial} \rho(\zeta)\left(L_{1}^{\prime}, \bar{L}_{2}^{\prime}\right) \\
& =\frac{\partial^{2} \rho}{\partial \zeta_{1} \partial \bar{\zeta}_{2}}(\zeta)+\mathcal{O}\left(\widetilde{b}_{1}(\zeta)+\widetilde{b}_{2}(\zeta)\right) .
\end{aligned}
$$

From (23), we see that

$$
\begin{aligned}
D_{1}\left(\frac{\partial^{2} \rho}{\partial \zeta_{1} \partial \bar{\zeta}_{2}}\right)\left(\widetilde{\zeta}^{\delta}\right) & =\mathcal{O}\left(\left|e_{\delta}\right|\right)=\mathcal{O}(\delta) \\
& =\frac{\partial^{2} \rho}{\partial \zeta_{1} \partial \bar{\zeta}_{2}}\left(\widetilde{\zeta}^{\delta}\right),
\end{aligned}
$$

and it follows from (61) that

$$
\left|\widetilde{D}_{2} \frac{\partial^{2} \rho}{\partial \zeta_{1} \partial \bar{\zeta}_{2}}\left(\widetilde{\zeta}^{\delta}\right)\right| \lesssim \delta_{s} \tau_{1}^{-1} \tau_{s}^{-2} .
$$

Therefore (80) follows from (73), (81), and (83) and by using Taylor series method.
Note that $T\left(\widetilde{z}^{\delta}, \sigma^{s} \delta\right):=t_{s}=q_{\nu(s)}$, for some $q_{\nu(s)}$, and hence there exist $j>0, k>0$ with $j+k=t_{s}$. In view of (79), we may assume that

$$
\left|L_{2}\left(\operatorname{Re} \mathscr{L}_{j-1, k} \partial \bar{\partial} r(z)\right)\right| \approx \delta_{s} \tau_{s}^{-t_{s}}, \quad z \in Q_{\gamma \delta}^{s}\left(\widetilde{z}^{\delta}\right),
$$

is valid (when $j=1$, we replace $\mathscr{L}_{j-1, k}$ by $\mathscr{L}_{j, k-1}$ ). Set

$$
G(z)=\operatorname{Re} \mathscr{L}_{j-1, k} \partial \bar{\partial} r(z) .
$$

By using the estimates (73)-(76), one obtains that

$$
\begin{aligned}
\left|L_{1} G\right| & \leq\left|D_{1} \frac{\partial^{t_{s}-1} \rho}{\partial \zeta_{2}^{j-1} \partial \bar{\zeta}_{2}^{k}}\right|+\left|D_{1} R_{j+k-1}\right|+\left|b_{1} G\right| \\
& \leq C_{2} \delta_{s} \tau_{1}^{-1} \tau_{s}^{-t_{s}+1},
\end{aligned}
$$

and similarly,

$$
\left|\partial \bar{\partial} G\left(L_{j}, \bar{L}_{k}\right)(z)\right| \leq C_{2} \delta_{s} \widetilde{\tau}_{j}^{-1} \widetilde{\tau}_{k}^{-1} \tau_{s}^{-t_{s}+1}, \quad j, k=1,2,
$$

for $z \in Q_{\gamma \delta}^{s}\left(\widetilde{z}^{\delta}\right)$, where $\widetilde{\tau}_{1}=\tau_{1}$ and $\widetilde{\tau}_{2}=\tau_{s}$.

Lemma 20. Assume that (59) holds. Then

$$
|G(z)| \leq C_{2} \sigma^{1 / t_{s}} \delta_{s} \tau_{s}^{-t_{s}+1}, \quad z \in Q_{\gamma \delta}^{s}\left(\widetilde{z}^{\delta}\right) .
$$

Proof. Suppose $z \in Q_{\gamma \delta}^{s}\left(\widetilde{z}^{\delta}\right)$. In view of (51)-(53), (56), and (59), we see that

$$
\begin{aligned}
\left(\frac{\sigma^{s+1} \delta}{A_{t_{s}-1}(z)}\right)^{1 /\left(t_{s}-1\right)} & \geq \tau\left(z, \sigma^{s+1} \delta\right)=\left(\frac{\sigma^{s+1} \delta}{A_{t_{s}}(z)}\right)^{1 / t_{s}} \\
& =\sigma^{1 / t_{s}} \tau\left(z, \sigma^{s} \delta\right) \approx \sigma^{1 / t_{s}} \tau_{s},
\end{aligned}
$$

and hence it follows that

$$
A_{t_{s}-1}(z) \lesssim \sigma^{1 / t_{s}} \delta_{s} \tau_{s}^{-t_{s}+1}
$$

This together with (73)-(78) implies the estimate (88).

In the sequel, we write

$$
L=a_{1} L_{1}+a_{2} L_{2}+a_{3} L_{3} .
$$

Lemma 21. There is a positive number $\sigma>0$, independent of $\widetilde{z}^{\delta}$ and $\delta$, such that if $z \in Q_{\gamma \delta}^{s}\left(\widetilde{z}^{\delta}\right)$ and if (59) holds, then there are constants $c_{2}>0$ and $C_{2}>0$, independent of $\tilde{z}, \delta$ and $\sigma>0$, such that

$$
\begin{aligned}
\partial \bar{\partial} G^{2}(L, \bar{L})(z) \geq & c_{2} \delta_{s}^{2} \tau_{s}^{-2 t_{s}}\left|a_{2}\right|^{2} \\
& -C_{2} \delta_{s}^{2} \tau_{1}^{-2} \tau_{s}^{-2 t_{s}+2}\left|a_{1}\right|^{2}-C_{2}\left|a_{3}\right|^{2} .
\end{aligned}
$$

Proof. Suppose $z \in Q_{\gamma \delta}^{s}\left(\widetilde{z}^{\delta}\right)$. From (87) and (88), we note that

$$
\left|G(z) \partial \bar{\partial} G(z)\left(L_{j}, \bar{L}_{k}\right)\right| \lesssim \sigma^{1 / t_{s}} \delta_{s}^{2} \tau_{s}^{-2 t_{s}+2} \widetilde{\tau}_{j}^{-1} \widetilde{\tau}_{k}^{-1},
$$


for $j, k=1,2$ where $\widetilde{\tau}_{1}=\tau_{1}$ and $\widetilde{\tau}_{2}=\tau_{s}$. Using (84)-(88) and (93) and by using small (large) constant method, one obtains that

$$
\begin{aligned}
\partial \bar{\partial} G(z)^{2}(L, \bar{L})=2|L G(z)|^{2}+2 G(z) \partial \bar{\partial} G(L, \bar{L})(z) \\
\geq 2 c_{2} \delta_{s}^{2} \tau_{s}^{-2 t_{s}}\left|a_{2}\right|^{2} \\
\quad+4 \operatorname{Re}\left(\sum_{1 \leq j<k \leq 3}\left(L_{j} G\right)\left(\bar{L}_{k} G\right) a_{j} \bar{a}_{k}\right) \\
\quad+2 \sum_{1 \leq j \leq k \leq 3} G(z) \partial \bar{\partial} G(z)\left(L_{j}, \bar{L}_{k}\right) a_{j} \bar{a}_{k} \\
\geq c_{2} \delta_{s}^{2} \tau_{s}^{-2 t_{s}}\left|a_{2}\right|^{2}-C_{2} \delta_{s}^{2} \tau_{1}^{-2} \tau_{s}^{-2 t_{s}+2}\left|a_{1}\right|^{2}-C_{2}\left|a_{3}\right|^{2},
\end{aligned}
$$

for some $c_{2}>0$ and $C_{2}>0$ provided $\sigma>0$ is sufficiently small.

Remark 22. From now on, we fix constants $c_{2}>0$ and $C_{2}>0$, which depend only on the derivatives of $r$ or $\rho$ of order up to $\eta$ on $V$, satisfying (69), (73), (80), and (86)-(92), and set $C_{2}=c_{2}^{-1}$ for a convenience. Now we choose and fix $\gamma>0$ and then fix $\sigma>0$ so that

$$
\begin{aligned}
40 C_{2}^{2} \gamma^{1 / 2} & \leq 1, \text { and } \\
420 C_{2}^{4} \gamma^{-7 / 2} \sigma^{2 / m} & \leq \frac{1}{16} .
\end{aligned}
$$

\section{Estimates on the Bergman Kernels}

Recall that $\widetilde{z}^{\delta}=\pi(z(\delta))=\left(d \delta^{1 / \eta}, 0, e_{\delta}\right) \in b \Omega$ where $z(\delta)=$ $\left(d \delta^{1 / \eta}, 0,-\delta\right)$ and where $\pi$ is the projection defined before (19). Also note that $\Phi_{\widetilde{z}^{\delta}}\left(\widetilde{\zeta}^{\delta}\right)=\widetilde{z}^{\delta}$ where $\widetilde{\zeta}^{\delta}=\left(d \delta^{1 / \eta}, 0,0\right)$ and where $\Phi_{\tilde{z}^{\delta}}$ is the holomorphic coordinate function defined in Proposition 11 about $\widetilde{z}=\widetilde{z}^{\delta}$. Also recall $C_{b}\left(z_{0}, \delta_{0}\right)$ defined in (20). In this section we estimate the Bergman kernel function $K_{\Omega}\left(z, z^{\delta}\right)$, for $z \in \Omega$ and $z^{\delta} \in C_{b}\left(z_{0}, \delta_{0}\right)$.

To get optimal estimates of the Bergman kernel, we need to construct a plurisubharmonic function which has maximal Hessian near each thin neighborhood of $b \Omega$ as in $[2,15]$. It contains complicated estimates depending on the type conditions of each boundary points. In this paper, however, we will construct such functions only at $\widetilde{z}^{\delta} \in b \Omega$. This will make the estimates much simpler than those in $[2,15]$ but still contain many complicated estimates.

Note that $\sigma>0$ and $\gamma>0$ are fixed in Remark 22 and hence the type $t_{s}$ and the integer $s$ defined in (59) depend only on $\widetilde{z}^{\delta} \in b \Omega$. Recall that $\delta_{s}=\sigma^{s} \delta, \tau_{1}=\delta^{1 / \eta}, \tau_{2}=\tau\left(\widetilde{z}^{\delta}, \delta\right)$, and $\tau_{s}=\tau\left(\widetilde{z}^{\delta}, \delta_{s}\right)$. From (54) we have

$$
\sigma^{s / 2} \tau_{2} \leq \tau_{s} \leq \sigma^{s / m} \tau_{2} .
$$

Let us write $L=a_{1} L_{1}+a_{2} L_{2}+a_{3} L_{3}$.

Proposition 23. There exist a smooth plurisubharmonic function $g_{z^{\delta}}$ on $\bar{\Omega}$ that satisfies the following: (i) $\left|g_{\tilde{z}^{\delta}}(z)\right| \leq 1$, for $z \in \bar{\Omega}$, and $g_{\tilde{z}^{\delta}}$ is supported in $Q_{\gamma \delta}^{s}\left(\widetilde{z}^{\delta}\right) \cap$ $\bar{\Omega}$.

(ii) There exist a small constant $b>0$ such that if $z \in$ $Q_{2 b \delta}\left(\widetilde{z}^{\delta}\right) \cap \bar{\Omega}$, then

$$
\partial \bar{\partial} g_{\tilde{z}^{\delta}}(L, \bar{L})(z) \approx \tau_{1}^{-2}\left|a_{1}\right|^{2}+\tau_{2}^{-2}\left|a_{2}\right|^{2}+\delta^{-2}\left|a_{3}\right|^{2} .
$$
then

(iii) If $\Phi_{\widetilde{z}^{\delta}}(\zeta)=\left(z_{1}, z_{2}, \Phi_{3}(\zeta)\right)$ where $\Phi_{3}$ is defined in (22),

$$
\left|\widetilde{D}^{\alpha}\left(g_{\widetilde{z}^{\delta}} \circ \Phi(\zeta)\right)\right| \leq C_{\alpha} \tau_{1}^{-\alpha_{1}} \tau_{2}^{-\alpha_{2}} \delta^{-\alpha_{3}} .
$$

holds for all $\zeta \in R_{\gamma \delta}^{s}\left(\widetilde{\zeta}^{\delta}\right)$ where $\widetilde{D}^{\alpha}=\widetilde{D}_{1}^{\alpha_{1}} \widetilde{D}_{2}^{\alpha_{2}} \widetilde{D}_{3}^{\alpha_{3}}$.

Proof. For each fixed $\widetilde{z}^{\delta}$, we note that the integers $s=s\left(\widetilde{z}^{\delta}\right)$ and $t_{s}$, defined in (59), will be fixed. Set $\widetilde{\tau}_{1}=\tau_{1}$ and $\widetilde{\tau}_{2}=\tau_{s}$. Note that $\gamma^{-2} \sigma^{-4 s} \widetilde{\tau}_{i}^{2} \leq 1$ provided $\delta>0$ is sufficiently small. Since $\delta_{s}=\sigma^{s} \delta$, it follows from (80) that

$$
\begin{aligned}
& \left|\partial \bar{\partial} r(z)\left(L_{1}, \bar{L}_{2}\right) a_{1} \bar{a}_{2}\right| \\
& \quad \leq C_{2} \gamma \delta\left(\tau_{1}^{-2}\left|a_{1}\right|^{2}+\sigma^{2 s} \tau_{s}^{-2}\left|a_{2}\right|^{2}\right), \text { and } \\
& \left|\partial \bar{\partial} r(z)\left(L_{i}, \bar{L}_{3}\right) a_{i} \bar{a}_{3}\right| \\
& \quad \leq C_{2} \gamma \sigma^{2 s} \delta \widetilde{\tau}_{i}^{-2}\left|a_{i}\right|^{2}+C_{2} \gamma^{-1} \sigma^{-2 s} \delta^{-1} \widetilde{\tau}_{i}^{2}\left|a_{3}\right|^{2} \\
& \quad \leq C_{2} \gamma \sigma^{2 s}\left(\delta \tilde{\tau}_{i}^{-2}\left|a_{i}\right|^{2}+\delta^{-1}\left|a_{3}\right|^{2}\right), \quad i=1,2,
\end{aligned}
$$

for $z \in Q_{\gamma \delta}^{s}\left(\widetilde{z}^{\delta}\right)$. From now on, we fix $\lambda=420 C_{2}^{2} \gamma^{-9 / 2}$ and set $\lambda_{s}=\sigma^{-2 s} \lambda$.

We may assume that the level sets of $r$ are pseudoconvex on $V$ and $\left|L_{3} r\right|^{2} \geq c_{0}^{2}>0$ on $V \cap \Omega$, where we may assume that $c_{0}^{2} \geq 4 c_{2}$. Also $4 C_{2} \gamma^{1 / 2} \leq c_{2} / 10$ by (95). Therefore it follows from (69) and (99) that

$$
\begin{aligned}
& \lambda_{s} \delta^{-1} \partial \bar{\partial} r(L, \bar{L})+\left(\lambda_{s} \delta^{-1}\right)^{2}|L r|^{2} \\
& \quad=\lambda_{s} \delta^{-1} \sum_{k=1}^{3} \partial \bar{\partial} r\left(L_{k}, \bar{L}_{k}\right)\left|a_{k}\right|^{2}+2 \lambda_{s} \delta^{-1} \\
& \quad \cdot \operatorname{Re} \sum_{1 \leq j<k \leq 3} \partial \bar{\partial} r\left(L_{j}, \bar{L}_{k}\right) a_{j} \bar{a}_{k}+\lambda_{s}^{2} \delta^{-2}\left|a_{3}\right|^{2}\left|L_{3} r\right|^{2} \\
& \geq \lambda_{s} \delta^{-1}\left[\frac{4 c_{2}}{5} \delta \tau_{1}^{-2}\left|a_{1}\right|^{2}\right. \\
& \left.\quad+\left(\partial \bar{\partial} r(z)\left(L_{2}, \bar{L}_{2}\right)-\frac{c_{2}}{10} \gamma^{1 / 2} \sigma^{2 s} \delta \tau_{s}^{-2}\right)\left|a_{2}\right|^{2}\right] \\
& \quad+3 c_{2} \lambda_{s}^{2} \delta^{-2}\left|a_{3}\right|^{2},
\end{aligned}
$$

for $z \in Q_{\gamma \delta}^{s}\left(\widetilde{z}^{\delta}\right)$.

Let $\psi(\zeta)$ be defined by

$$
\psi(\zeta)=\chi\left(\tau_{1}^{-2}\left|\zeta_{1}-d \delta^{1 / \eta}\right|^{2}+\tau_{s}^{-2}\left|\zeta_{2}\right|^{2}+\delta_{s}^{-2}\left|\zeta_{3}\right|^{2}\right),
$$

where $\chi$ is a smooth function such that $\chi(t)=1$ for $t<\gamma^{2} / 9$ and $\chi(t)=0$ for $t \geq \gamma^{2}$, satisfying $\left|D^{k} \chi\right| \leq C_{k} \gamma^{-2 k}$. Set $\Psi(z)=$ 
$\psi\left(\left(\Phi_{\widetilde{z}^{\delta}}\right)^{-1}(z)\right)$. Note that $\Phi_{\widetilde{z}^{\delta}}^{-1}(z)$ has similar expression as in (22). Thus it follows, from (22), (29), (30), and chain rule, that

$$
\left|D^{\alpha} \Psi(z)\right| \leq C_{|\alpha|} \gamma^{-2|\alpha|} \tau_{1}^{-\alpha_{1}} \tau_{s}^{-\alpha_{2}} \delta_{s}^{-\alpha_{3}} \quad z \in Q_{\gamma \delta}^{s}\left(\widetilde{z}^{\delta}\right) .
$$

Here $\alpha=\left(\alpha_{1}, \alpha_{2}, \alpha_{3}\right)$ and $|\alpha|=\alpha_{1}+\alpha_{2}+\alpha_{3}$. Since $C_{2}=c_{2}^{-1}$, one obtains

$$
\begin{aligned}
& |\partial \bar{\partial} \Psi(z)(L, \bar{L})| \\
& \leq C_{2} \gamma^{-4}\left(\tau_{1}^{-2}\left|a_{1}\right|^{2}+\tau_{s}^{-2}\left|a_{2}\right|^{2}+\delta_{s}^{-2}\left|a_{3}\right|^{2}\right), \\
& \lambda_{s} \delta^{-1}\left|L_{i} \Psi(z)\right|\left|a_{i}\right|\left|a_{3}\right| \\
& \quad \leq 10 C_{2} \gamma^{-4} \widetilde{\tau}_{i}^{-2}\left|a_{i}\right|^{2}+\frac{c_{2}}{10} \lambda_{s}^{2} \delta^{-2}\left|a_{3}\right|^{2}, \quad i=1,2,
\end{aligned}
$$

where $\widetilde{\tau}_{1}=\tau_{1}$ and $\widetilde{\tau}_{2}=\tau_{s}$.

Suppose that $z$ satisfies $\Psi(z) \geq 1 / 4$. Using the fact that $L_{k} r=0, k=1,2$, and the fact that $84 C_{2} \gamma^{-9 / 2}=\left(c_{2} / 5\right) \lambda \leq$ $\left(c_{2} / 5\right) \lambda_{s}$, it follows from (100)-(103) that

$$
\begin{aligned}
& \partial \bar{\partial}\left(\Psi e^{\lambda_{s} \delta^{-1} r}\right)(L, \bar{L})=e^{\lambda_{s} \delta^{-1} r}[\partial \bar{\partial} \Psi(L, \bar{L}) \\
& \left.\quad+2 \lambda_{s} \delta^{-1} \sum_{i=1}^{3} \operatorname{Re}\left(\left(L_{i} \Psi\right)\left(\bar{L}_{3} r\right)\right) a_{i} \bar{a}_{3}\right] \\
& \quad+e^{\lambda_{s} \delta^{-1} r}\left[\lambda_{s} \delta^{-1} \Psi \partial \bar{\partial} r(L, \bar{L})+\lambda_{s}^{2} \delta^{-2} \Psi|L r|^{2}\right] \geq \frac{1}{4} \\
& \quad \cdot e^{\lambda_{s} \delta^{-1} r}\left[\frac{3 c_{2}}{5} \lambda_{s} \tau_{1}^{-2}\left|a_{1}\right|^{2}+c_{2} \lambda_{s}^{2} \delta^{-2}\left|a_{3}\right|^{2}\right]+\frac{1}{4} \\
& \quad \cdot e^{\lambda_{s} \delta^{-1} r}\left[\lambda_{s} \delta^{-1} \partial \bar{\partial} r(z)\left(L_{2}, \bar{L}_{2}\right)-\frac{2 c_{2}}{5} \gamma^{1 / 2} \lambda \tau_{s}^{-2}\right] \\
& \quad \cdot\left|a_{2}\right|^{2} \cdot
\end{aligned}
$$

We note that the negative part in (104) contains $\gamma^{1 / 2} \lambda$ instead of $\gamma^{1 / 2} \lambda_{s}$.

Let $h$ be a smooth convex function such that $h(t)=0$ for $t \leq 1 / 2$ and $h(t)>0$ for $t>1 / 2$ and $h(9 / 8) \leq 1$. Set $G_{\widetilde{z}^{\delta}}(z)=\Psi(z) e^{\lambda_{s} \delta^{-1} r(z)}$ and set $g_{\widetilde{z}^{\delta}}(z)=h\left(G_{\widetilde{z}^{\delta}}(z)\right)$. Suppose $T\left(\widetilde{z}^{\delta}, \delta\right)=2$. Then $s=0$, and hence (79) holds for $\delta_{s}=\delta$ with $j=k=1$; that is,

$$
c_{2} \delta \tau_{2}^{-2} \leq \partial \bar{\partial} r(z)\left(L_{2}, \bar{L}_{2}\right) \leq C_{2} \delta \tau_{2}^{-2}, \quad z \in Q_{\gamma \delta}\left(\widetilde{z}^{\delta}\right) .
$$

For those $z$ with $\Psi(z) \geq 1 / 4$, it follows from (104) (with $\lambda_{s}=$ $\lambda$ ) and (105) that

$$
\begin{aligned}
& \partial \bar{\partial} G_{\widetilde{z}^{\delta}}(z) \\
& \quad \geq \frac{3 c_{2} \lambda}{20} e^{\lambda \delta^{-1} r}\left[\tau_{1}^{-2}\left|a_{1}\right|^{2}+\tau_{2}^{-2}\left|a_{2}\right|^{2}+\delta^{-2}\left|a_{3}\right|^{2}\right] .
\end{aligned}
$$

If $\Psi(z) \leq 1 / 4$, then $G_{\widetilde{z}^{\delta}}(z) \leq 1 / 4$ and hence $g_{\widetilde{z}^{\delta}}(z)=0$. Hence $g_{\widetilde{z}^{\delta}}$ is a smooth plurisubharmonic function supported on $Q_{\gamma \delta}\left(\widetilde{z}^{\delta}\right)$, and $\left|g_{\tilde{z}^{\delta}}\right| \leq 1$.
Now assume $T\left(\widetilde{z}^{\delta}, \delta\right)>2$ and assume that (59) holds. Then (79) holds for some positive integers $j, k$ with $j+k=t_{s}$. Let $G(z)$ be the function defined in (85). From (88) and (95), we see that

$$
\begin{aligned}
\lambda \gamma^{1 / 2} \delta_{s}^{-2} \tau_{s}^{2 t_{s}-2} G(z)^{2} & \leq \lambda \gamma^{1 / 2} C_{2}^{2} \sigma^{2 / t_{s}} \\
& \leq 420 C_{2}^{4} \gamma^{-7 / 2} \sigma^{2 / m} \leq \frac{1}{16}
\end{aligned}
$$

$z \in Q_{\gamma \delta}^{s}\left(\widetilde{z}^{\delta}\right)$, because $\lambda=420 C_{2}^{2} \gamma^{-4}$ and $t_{s} \leq m$. Set

$$
\begin{aligned}
& g_{\widetilde{z}^{\delta}}(z) \\
& \quad=h\left(\Psi(z) e^{\lambda_{s} \delta^{-1} r(z)}+\phi\left(\lambda \gamma^{1 / 2} \delta_{s}^{-2} \tau_{s}^{2 t_{s}-2} G(z)^{2}\right)\right),
\end{aligned}
$$

where $\phi(t)$ is a smooth function that satisfies $\phi(t)=t$, for $t \leq 1 / 16, \phi(t)=0$ for $t \geq 1$, and $\phi(t) \leq 1 / 8$ for all $t$. Thus $g_{\widetilde{z}^{\delta}} \in C_{0}^{\infty}\left(Q_{\gamma \delta}^{s}\left(\widetilde{z}^{\delta}\right)\right)$ and $\left|g_{\widetilde{z}^{\delta}}\right| \leq 1$ because $h(9 / 8) \leq 1$. By (107) we note that $\phi(z)=z$ on $Q_{\gamma \delta}^{s}\left(\widetilde{z}^{\delta}\right)$, and we also note that $g_{\widetilde{z}^{\delta}}=0$ if $\Psi(z) e^{\lambda_{s} \delta^{-1} r(z)} \leq 3 / 8$, in particular, $g_{\widetilde{z}^{\delta}}=0$ outside $Q_{\gamma \delta}^{s}\left(\widetilde{z}^{\delta}\right)$. From (92), we obtain that

$$
\begin{aligned}
& \lambda \gamma^{1 / 2} \delta_{s}^{-2} \tau_{s}^{2 t_{s}-2} \partial \bar{\partial} G(z)^{2}(L, \bar{L}) \\
& \geq \gamma^{1 / 2}\left(c_{2} \lambda \tau_{s}^{-2}\left|a_{2}\right|^{2}-C_{2} \lambda \tau_{1}^{-2}\left|a_{1}\right|^{2}-C_{2} \lambda \delta_{s}^{-2}\left|a_{3}\right|^{2}\right) \\
& \geq c_{2} \gamma^{1 / 2} \lambda \tau_{s}^{-2}\left|a_{2}\right|^{2}-\frac{c_{2}}{40} \lambda \tau_{1}^{-2}\left|a_{1}\right|^{2}-\frac{c_{2}}{40} \lambda \delta_{s}^{-2}\left|a_{3}\right|^{2}
\end{aligned}
$$

for $z \in Q_{\gamma \delta}^{s}\left(\widetilde{z}^{\delta}\right)$, because $\gamma^{1 / 2} C_{2} \leq c_{2} / 40$.

Assuming that $\Psi(z) e^{\lambda_{s} \delta^{-1} r(z)} \geq 3 / 8$, we note that the negative coefficient part of $\left|a_{2}\right|^{2}$ of the Hessian of $\Psi(z) e^{\lambda_{s} \delta^{-1} r(z)}$ in (104) is controlled by the first term in the third line of (109), and the error terms of the coefficients of $\left|a_{1}\right|^{2}$ and $\left|a_{3}\right|^{2}$ in the third line of (109) are controlled by the corresponding coefficients of the Hessian of $\Psi(z) e^{\lambda_{s} \delta^{-1} r(z)}$ in (104). In either $T\left(\widetilde{z}^{\delta}, \delta\right)=2$ or $T\left(\widetilde{z}^{\delta}, \delta\right)>2$ cases, it follows from (104), (106), and (109) that

$$
\begin{gathered}
\partial \bar{\partial} g_{\widetilde{z}^{\delta}}(L, \bar{L}) \geq \frac{c_{2}}{32} e^{\lambda_{s} \delta^{-1} r(z)}\left(\lambda_{s} \tau_{1}^{-2}\left|a_{1}\right|^{2}\right. \\
\left.+\gamma^{1 / 2} \lambda \tau_{s}^{-2}\left|a_{2}\right|^{2}+\lambda_{s}^{-2} \delta^{-2}\left|a_{3}\right|^{2}\right)
\end{gathered}
$$

for $z \in Q_{\gamma \delta}^{s}\left(\widetilde{z}^{\delta}\right)$.

Note that parameters, $c_{2}, C_{2}, \gamma, \sigma$, and $\lambda$, are fixed in Remark 22, independent of $\delta>0$. Therefore the upper bound of $g_{\widetilde{z}^{\delta}}$ follows from (84)-(88), (96), (99), (102), and (103). Note that $e^{\lambda_{s} \delta^{-1} r(z)}>e^{-1 / 4}>3 / 4$, if $r(z)>-\delta / 4 \lambda_{s}=-\delta \sigma^{2 s} / 4 \lambda$, and this property holds on $Q_{2 b \delta}\left(\widetilde{z}^{\delta}\right)$ if we take $b>0$ sufficiently small; say, $0<2 b<\sigma^{2 m} / \lambda^{2}$. Also note that $\Psi=1$ on $Q_{2 b \delta}\left(\widetilde{z}^{\delta}\right)$. This fact together with (96) and (110) proves properties (i) and (ii). Property (iii) follows from (22), (30), (32), and (96).

For each $z^{\delta}=\left(d \delta^{1 / \eta}, 0, e_{\delta}-b \delta\right) \in C_{b}\left(z_{0}, \delta_{0}\right)$, set $\zeta^{\delta}:=$ $\Phi_{\widetilde{z}^{\delta}}^{-1}\left(z^{\delta}\right)=\left(d \delta^{1 / \eta}, 0,-b \delta\right)$. 
Proposition 24. There is a small constant $a>0$ such that $R_{2 a \delta}\left(\zeta^{\delta}\right) \subset \subset \Omega$ for all sufficiently small $\delta>0$.

Proof. From (22)-(29), we obtain that

$$
\rho\left(\zeta^{\delta}\right)=r\left(z^{\delta}\right)=-b \delta+\mathcal{O}\left(\delta^{1+1 / \eta}\right)<-\frac{b \delta}{2}
$$

for all sufficiently small $\delta>0$. Assume $\zeta \in R_{2 a \delta}\left(\zeta^{\delta}\right)$ and write

$$
\begin{aligned}
\rho(\zeta)= & {\left[\rho(\zeta)-\rho\left(d \delta^{1 / \eta}, \zeta_{2}, \zeta_{3}\right)\right] } \\
& +\left[\rho\left(d \delta^{1 / \eta}, \zeta_{2}, \zeta_{3}\right)-\rho\left(\zeta^{\delta}\right)\right]+\rho\left(\zeta^{\delta}\right) \\
:= & E_{1}+E_{2}+\rho\left(\zeta^{\delta}\right) .
\end{aligned}
$$

From (61), and by using Taylor series method, one obtains that

$$
\left|E_{1}\right| \leq a \max _{\left|\widetilde{\zeta}_{1}-d \delta^{1 / \eta}\right|<2 a \delta^{1 / \eta}}\left|D_{1} \rho\left(\widetilde{\zeta}_{1}, \zeta_{2}, \zeta_{3}\right)\right| \delta^{1 / \eta} \leq 2 a C_{2} \delta
$$

for a uniform constant $C_{2}>0$. Similarly, we obtain $\left|E_{2}\right| \leq$ $4 a C_{2} \delta$. Combining these estimates and (111) and if we set $a=$ $b / 24 C_{2}$, then we obtain that

$$
\rho(\zeta)<6 a C_{2} \delta-\frac{b \delta}{2}=-\frac{b \delta}{4}, \quad \zeta \in R_{a \delta}\left(\zeta^{\delta}\right) .
$$

Remark 25. (1) Set $\tilde{g}_{\delta}(\zeta):=g_{\tilde{z}^{\delta}} \circ \Phi_{\widetilde{z}^{\delta}}(\zeta)$. Then, by functoriality, Proposition 23 holds, where $g_{\tilde{z}^{\delta}}$ is replaced by $\tilde{g}_{\delta}$, and $Q_{\gamma \delta}\left(\widetilde{z}^{\delta}\right)$ is replaced by $R_{\gamma \delta}\left(\widetilde{\zeta}^{\delta}\right)$.

For each fixed $\delta>0$, and for each fixed $\widetilde{z}^{\delta}=\left(d \delta^{1 / \eta}\right.$, $\left.0, e_{\delta}\right) \in b \Omega$, set $\Omega_{\widetilde{z}^{\delta}}=\Phi_{\widetilde{z}^{\delta}}^{-1}(\Omega)$. Note that $\left|\operatorname{det}\left(J_{\mathbb{C}} \Phi_{\widetilde{z}^{\delta}}^{-1}(z)\right)\right|=$ $2\left|\left(\partial r / \partial z_{3}\right)\left(\widetilde{z}^{\delta}\right)\right| \geq 2 c_{0}>0$ on $V$. Thus it follows, from transformation formula, that

$$
K_{\Omega}\left(z, z^{\delta}\right)=4\left|\frac{\partial r}{\partial z_{3}}\left(\widetilde{z}^{\delta}\right)\right|^{2} K_{\Omega_{z^{\delta}}}\left(\zeta, \zeta^{\delta}\right) .
$$

In view of Propositions 23 and 24, there is a smooth plurisubharmonic weight function $g_{\tilde{z}^{\delta}}$ which has maximal Hessian on $Q_{a \delta}\left(z^{\delta}\right) \subset \subset \Omega$. We also note that $\tau\left(\widetilde{z}^{\delta}, \delta\right) \approx \tau\left(z^{\delta}, \delta\right)$ by (56). If we use these properties and (115), we get the following estimates for the Bergman kernel function $K_{\Omega}\left(z^{\delta}, z^{\delta}\right)$ at $z^{\delta} \in$ $C_{b}\left(z_{0}, \delta_{0}\right)$ as in Theorem 6.1 in [2]:

$$
\begin{aligned}
& K_{\Omega}\left(z^{\delta}, z^{\delta}\right) \approx \delta^{-2} \delta^{-2 / \eta} \tau\left(z^{\delta}, \delta\right)^{-2}, \\
& z^{\delta} \in C_{b}\left(z_{0}, \delta_{0}\right) .
\end{aligned}
$$

This proves Theorem 2 .

Now we want to get derivative estimates of $K\left(z, z^{\delta}\right)$ for $z \in \Omega$ and $z^{\delta} \in C_{b}\left(z_{0}, \delta_{0}\right)$. In view of (115), we will estimate $K_{\Omega_{z^{\delta}}}\left(\zeta, \zeta^{\delta}\right)$ where $z=\Phi_{\widetilde{z}^{\delta}}(\zeta)$ and $z^{\delta}=\Phi_{\widetilde{z}^{\delta}}\left(\zeta^{\delta}\right)$. We will follow the methods in $[3,9]$ which use dilated coordinates.
For each fixed $\delta>0$, we recall that $\tau_{1}=\delta^{1 / \eta}, \tau_{2}=\tau\left(z^{\delta}, \delta\right)$ and $\tau_{3}=\delta$. Define a dilation map $D_{\delta}$ given by

$$
\begin{aligned}
D_{\delta}(\zeta) & =\left(\frac{\zeta_{1}-d \delta^{1 / \eta}}{a \tau_{1}}, \frac{\zeta_{2}}{a \tau_{2}}, \frac{\zeta_{3}+b \delta}{a \tau_{3}}\right):=\left(w_{1}, w_{2}, w_{3}\right) \\
& =w
\end{aligned}
$$

set

$$
\begin{aligned}
\rho_{\delta}(w) & :=\delta^{-1}\left(\rho \circ D_{\delta}^{-1}(w)\right), \\
\Omega_{\delta} & =\left\{w \in \mathbb{C}^{3} ; \rho_{\delta}(w)<0\right\},
\end{aligned}
$$

and set

$$
\lambda_{\delta}(w):=\tilde{g}_{\delta} \circ D_{\delta}^{-1}(w),
$$

where $\tilde{g}_{\delta}(\zeta):=g_{\widetilde{z}^{\delta}} \circ \Phi_{\widetilde{z}^{\delta}}(\zeta)$ and where $g_{\widetilde{z}^{\delta}}$ is defined in Proposition 23 .

Set

$$
\begin{aligned}
& L_{3}^{\delta}=\frac{\partial}{\partial w_{3}}, \\
& L_{k}^{\delta}=\frac{\partial}{\partial w_{k}}-\left(\frac{\partial \rho_{\delta}}{\partial w_{3}}\right)^{-1} \frac{\partial \rho_{\delta}}{\partial w_{k}} \frac{\partial}{\partial w_{3}}, \\
& k=1,2,
\end{aligned}
$$

and write $L^{\delta}=b_{1} L_{1}^{\delta}+b_{2} L_{2}^{\delta}+b_{3} L_{3}^{\delta}$. The properties of $\lambda_{\delta}(w)$, which follow from Propositions 23 and 24 and Remark 25, are summarized in the following proposition.

Proposition 26. For each $\delta>0$ there is $\lambda_{\delta}(w)$, defined on $\Omega_{\delta}$, such that

(1) $\lambda_{\delta}(w)$ is smooth plurisubharmonic in $\Omega_{\delta}$, and $\left|\lambda_{\delta}\right| \leq 1$;

(2) $\operatorname{supp} \lambda_{\delta}(w) \subset P(0, \widetilde{C})$, for some $\widetilde{C}=2 a^{-1} \gamma>1$;

(3) $\partial \bar{\partial} \lambda_{\delta}\left(L^{\delta}, \bar{L}^{\delta}\right)(w) \approx\left|b_{1}\right|^{2}+\left|b_{2}\right|^{2}+\left|b_{3}\right|^{2}$ if $w \in P(0,1)$;

(4) $\left|D_{w}^{\alpha} \lambda_{\delta}(w)\right| \leq C_{\alpha}$.

The weight function with the properties in Proposition 26 is the key ingredient for the derivative estimates of the Bergman kernel function off the diagonal. Set $P=P(0, \widetilde{C})$ and let $N_{\delta}$ be the Neumann operator on $\Omega_{\delta}$. Then we have the following $L^{2}$ estimates of $N_{\delta}$ (Proposition 3.14 in [3]).

Proposition 27. Let $h \in L^{2}$ be a $(0,1)$ form and supp $h \subset P$. Then there is $C>0$, independent of $\delta>0$, so that

$$
\int_{\Omega_{\delta} \cap P}\left|N_{\delta} h\right|^{2} \leq C\|h\|^{2}
$$

Note that $D_{\delta}\left(\zeta^{\delta}\right)=0$. Set

$$
P(0, r):=\left\{w=\left(w_{1}, w_{2}, w_{3}\right):\left|w_{k}\right| \leq r, k=1,2,3\right\} .
$$

From (117) and Proposition 24, we note that

$$
D_{\delta}\left(R_{a \delta}\left(\zeta^{\delta}\right)\right)=P(0,1) \subset \subset P(0,2) \subset \subset \Omega_{\delta},
$$


independent of $\delta>0$. Let $\xi_{1}, \xi_{2} \in C_{0}^{\infty}(P(0,1))$ with $\xi_{1}=1$ in a neighborhood of 0 and $\xi_{2}=1$ on $\operatorname{supp} \xi_{1}$. From (123), we see that supp $\xi_{2} \subset P(0,1) \subset \subset P(0,2) \subset \subset \Omega_{\delta}$, independent of $\delta>0$. Therefore we have the following elliptic estimates:

$$
\begin{aligned}
\left\|\xi_{1} f\right\|_{s+2}^{2} \leq C_{s}\left(\left\|\xi_{2} \square_{\delta} f\right\|_{s}^{2}+\|f\|^{2}\right), & \\
& s \geq 0, f \in \operatorname{Dom}(\bar{\partial}) \cap \operatorname{Dom}\left(\bar{\partial}^{*}\right),
\end{aligned}
$$

where $\square_{\delta}$ is the complex Laplacian on $\Omega_{\delta}$.

Remark 28. The estimates in (124) are on the polydisc $P(0,1) \subset \subset P(0,2) \subset \subset \Omega_{\delta}$, strictly inside of $\Omega_{\delta}$, independent of $\delta>0$. Therefore we gain two derivatives in (124) and it is stable; that is, $C_{s}$ is independent of $\delta>0$. Also we note that we do not require that $\Delta_{1}\left(z_{0}\right)<\infty$. Since $P(0,2) \subset \subset P=P(0, \widetilde{C})$ where $\widetilde{C}=2 a^{-1} \gamma>2$, we can also apply the estimate (121) on $P(0,2)$.

Let $\phi \in C_{0}^{\infty}(P(0,1)), \int \phi=1$, and $\phi$ be polyradial. In terms of $w$-coordinates in (117), we have the following well known representation of Bergman kernel function on $\Omega_{\delta}$.

$$
K_{\Omega_{\delta}}(w, 0)=\phi(w)-\bar{\partial}_{*} N_{\delta} \bar{\partial} \phi(w) .
$$

Let $\chi \in C^{\infty}\left(\Omega_{\delta}\right)$ with $\chi=1$ outside $P(0,1)$ and $\chi=0$ on supp $\phi$. Combining (121)-(125), we can prove the following lemma as in the proof of Theorem 4.2 in [3].

Lemma 29. For each $s \geq 0$ there is $C_{s}>0$ such that

$$
\left\|\chi N_{\delta} \bar{\partial} \phi\right\|_{s} \leq C_{s}
$$

Now, if we use the estimate (126) with $s=|\alpha|+3$, we can prove Theorem 3 as in the proof of Theorem 4.2 in [3].

\section{Appendix}

We recall Herbort's example $\Omega_{H}$ in (6). Therefore $\eta=6=$ $\Delta_{1}(0)$ and hence $\tau_{1}=\delta^{1 / 6}, \tau_{2}=\delta^{1 / 3}$, and $\tau_{3}=\delta$ in our notations. For each fixed $\delta>0$, set $z^{\delta}=\left(\delta^{1 / 6} / 2,0,-\delta\right)$. Then $z^{\delta} \in \Omega_{H}$ and approaches to $0 \in b \Omega_{H}$ in "almost tangential direction" as the points do along $C_{b}\left(z_{0}, \delta_{0}\right)$. In this case, we will show that

$$
K_{\Omega_{H}}\left(z^{\delta}, z^{\delta}\right) \approx \delta^{-3}=\delta^{-2} \tau_{1}^{-2} \tau_{2}^{-2},
$$

which is exactly same result as Theorem 2 .

Proof of (A.1). Set

$$
\begin{aligned}
& P_{\delta}\left(z^{\delta}\right):=\left\{z:\left|z_{1}-\frac{\delta^{1 / 6}}{2}\right|<\frac{\delta^{1 / 6}}{10},\left|z_{2}\right|\right. \\
& \left.\quad<\frac{\delta^{1 / 3}}{10},\left|z_{3}+\delta\right|<\frac{\delta}{10}\right\} .
\end{aligned}
$$

Then the polydisc $P_{\delta}\left(z^{\delta}\right)$ about $z^{\delta}$ is contained in $\Omega_{H}$. Therefore the upper bound $K_{\Omega_{H}}\left(z^{\delta}, z^{\delta}\right) \lesssim \delta^{-3}$ follows. Let us show lower bounds.
Let $\Delta_{3}$ be the unit polydisc in $\mathbb{C}^{3}$. Since the localization lemma is valid for $\Omega_{H}$, we will estimate $K_{\Omega_{H} \cap \Delta_{3}}\left(z^{\delta}, z^{\delta}\right)$. Set

$$
\begin{aligned}
& P_{1}\left(z_{1}, z_{2}\right)=\left|z_{1}\right|^{6}+\left|z_{1}\right|^{2}\left|z_{2}\right|^{2}+\left|z_{2}\right|^{6}, \text { and } \\
& P_{2}\left(z_{1}, z_{2}\right)=\left|z_{1}\right|^{6}+\left|z_{1}\right|^{2}\left|z_{2}\right|^{2}
\end{aligned}
$$

and set

$$
\begin{aligned}
& G_{1}=\left\{\operatorname{Re} z_{3}+P_{1}\left(z_{1}, z_{2}\right)<0\right\}, \text { and } \\
& G_{2}=\left\{\operatorname{Re} z_{3}+P_{2}\left(z_{1}, z_{2}\right)<0\right\} .
\end{aligned}
$$

Then $\Omega_{H}=G_{1} \subset G_{2}$ and $z^{\delta} \in G_{1} \cap G_{2}$. Set $f_{\delta}=8 \delta^{11 / 6} z_{1} /\left(z_{3}-\right.$ $\delta)^{2}$. Then $f_{\delta}\left(z^{\delta}\right)=1$. Note that

$$
\begin{aligned}
& \left\|f_{\delta}\right\|_{L^{2}\left(G_{1} \cap \Delta_{3}\right)}^{2} \leq\left\|f_{\delta}\right\|_{L^{2}\left(G_{2} \cap \Delta_{3}\right)}^{2} \\
& \quad=16 \delta^{11 / 3} \int_{\left|z_{1}\right|,\left|z_{2}\right|<1}\left|z_{1}\right|^{2} \\
& \quad \cdot\left[\int_{\operatorname{Re} z_{3}<-P_{2}}\left|z_{3}-\delta\right|^{-4} d V_{2}\left(z_{3}\right)\right] d V_{4} \\
& \quad \leq \delta^{11 / 3} \int_{\left|z_{1}\right|,\left|z_{2}\right|<1}\left|z_{1}\right|^{2}\left(\delta+P_{2}\right)^{-2} d V_{4}:=(*),
\end{aligned}
$$

where we have used $\int_{\mathbb{R}}\left(d s /\left(1+s^{2}\right)^{2}\right)=c_{1}<\infty$. Set $z_{1}=\delta^{1 / 6} z_{1}^{\prime}$ and $z_{2}=\delta^{1 / 3} z_{2}^{\prime}$. Then

$$
\begin{aligned}
(*) & \lesssim \delta^{3} \int_{\left|z_{2}^{\prime}\right|<\delta^{-1 / 3}} \int_{\left|z_{1}^{\prime}\right|<\delta^{-1 / 6}}\left|z_{1}^{\prime}\right|^{2}(1 \\
& \left.+P_{2}\left(z_{1}^{\prime}, z_{2}^{\prime}\right)\right)^{-2} d V_{4}\left(z_{1}^{\prime}, z_{2}^{\prime}\right) \\
& \lesssim \delta^{3} \int_{0}^{\delta^{-1 / 3}} \int_{0}^{\delta^{-1 / 6}} r_{1}^{3} r_{2}\left(1+r_{1}^{2} r_{2}^{2}\right. \\
& \left.+r_{1}^{6}\right)^{-2} d r_{1} d r_{2}:=(* *)
\end{aligned}
$$

where we have used the polar coordinates: $z_{k}^{\prime}=r_{k} \sigma^{i \theta}, k=1,2$ in the second line.

$$
\begin{aligned}
& \text { Set } r_{1}^{4}=x \text { and } r_{2}^{2}=y \text {. Then } \\
& \begin{aligned}
(* *) & \leqslant \delta^{3} \int_{0}^{\delta^{-2 / 3}} \int_{0}^{\delta^{-2 / 3}}\left(1+x^{1 / 2} y+x^{3 / 2}\right)^{-2} d y d x \\
& =\delta^{7 / 3} \int_{0}^{\delta^{-2 / 3}} \frac{d x}{\left(1+x^{3 / 2}\right)\left(1+\delta^{-2 / 3} x^{1 / 2}+x^{3 / 2}\right)} \\
& :=\delta^{7 / 3} I(\delta) .
\end{aligned}
\end{aligned}
$$

Write $I(\delta)=\int_{0}^{1}+\int_{1}^{\delta^{-2 / 3}}:=I_{1}(\delta)+I_{2}(\delta)$. Set $\delta^{-2 / 3} x^{1 / 2}=x^{\prime}$. Then

$$
I_{1}(\delta) \lesssim \int_{0}^{\delta^{-2 / 3}} \frac{\delta^{4 / 3} x^{\prime} d x^{\prime}}{1+x^{\prime}} \leq \delta^{4 / 3} \int_{0}^{\delta^{-2 / 3}} d x^{\prime}=\delta^{2 / 3}
$$


Also,

$$
\begin{aligned}
I_{2}(\delta) & \leqslant \int_{1}^{\delta^{-2 / 3}} \frac{d x}{x^{3 / 2}\left(x^{3 / 2}+\delta^{-2 / 3} x^{1 / 2}\right)} \\
& \leq \delta^{2 / 3} \int_{1}^{\delta^{-2 / 3}} x^{-2} d x \leq \delta^{2 / 3} .
\end{aligned}
$$

Combining (A.5)-(A.9), we obtain that $\left\|f_{\delta}\right\|_{L^{2}\left(\Omega_{H}\right)}^{2} \lesssim \delta^{3}$. Therefore $K_{\Omega_{H}}\left(z^{\delta}, z^{\delta}\right) \gtrsim \delta^{-3}$.

Remark 30. Set $f(z)=\exp \left(z_{3} /\left(1-z_{3}\right)\right)$. Then $f$ is a peak function that peaks at $0 \in b \Omega_{H}$ for the domain $\Omega_{H}$.

\section{Data Availability}

No data were used to support this study.

\section{Conflicts of Interest}

The author declares that there are no conflicts of interest regarding the publication of this paper.

\section{Acknowledgments}

The author was partially supported by the Sogang University Research Fund.

\section{References}

[1] S. Bergman, The kernel function and conformal mapping, American Mathematical Society, Providence, R.I., 2nd edition, 1970.

[2] D. W. Catlin, "Estimates of invariant metrics on pseudoconvex domains of dimension two," Mathematische Zeitschrift, vol. 200, no. 3, pp. 429-466, 1989.

[3] J. D. McNeal, "Boundary behavior of the Bergman kernel function in C2," Duke Mathematical Journal, vol. 58, no. 2, pp. 499-512, 1989.

[4] A. Nagel, J.-P. Rosay, E. M. Stein, and S. Wainger, "Estimates for the Bergman and Szegö kernels in C2," Annals of Mathematics: Second Series, vol. 129, no. 1, pp. 113-149, 1989.

[5] J. D. McNeal, "Local geometry of decoupled pseudoconvex domains," in Complex analysis (Wuppertal, 1991), Aspects Math., E17, pp. 223-230, Friedr. Vieweg, Braunschweig, 1991.

[6] J. D. McNeal, "Estimates on the Bergman kernels of convex domains," Advances in Mathematics, vol. 109, no. 1, pp. 108-139, 1994.

[7] K. Diederich, G. Herbort, and T. Ohsawa, "The Bergman kernel on uniformly extendable pseudoconvex domains," Mathematische Annalen, vol. 273, no. 3, pp. 471-478, 1986.

[8] S. Cho, "Boundary behavior of the Bergman kernel function on some pseudoconvex domains in C," Transactions of the American Mathematical Society, vol. 345, no. 2, pp. 803-817, 1994.

[9] S. Cho, "Estimates of the Bergman kernel function on certain pseudoconvex domains in Cn," Mathematische Zeitschrift, vol. 222, no. 2, pp. 329-339, 1996.

[10] P. Charpentier and Y. Dupain, "Estimates for the Bergman and Szegö projections for pseudoconvex domains of finite type with locally diagonalizable Levi form," Publicacions Matemàtiques, vol. 50, no. 2, pp. 413-446, 2006.
[11] P. Charpentier, Y. Dupain, and M. Mounkaila, "Estimates weighted Bergman projections on pseudo-convex domains of finite type in Cn," Complex Variables and Elliptic Equations, vol. 59, no. 8, pp. 1070-1095, 2014.

[12] P. Charpentier, Y. Dupain, and M. Mounkaila, "On estimates for weighted Bergman projections," Proceedings of the American Mathematical Society, vol. 143, no. 12, pp. 5337-5352, 2015.

[13] J. P. D’Angelo, "Real hypersurfaces, orders of contact, and applications," Annals of Mathematics, vol. 115, no. 3, pp. 615-637, 1982.

[14] G. Herbort, "Logarithmic growth of the Bergman kernel for weakly pseudoconvex domains in C3 of finite type," Manuscripta Mathematica, vol. 45, no. 1, pp. 69-76, 1983.

[15] D. Catlin, "Subelliptic estimates for the $\partial$-Neumann problem on pseudoconvex domains," Annals of Mathematics: Second Series, vol. 126, no. 1, pp. 131-191, 1987.

[16] Y. You, "Necessary conditions for Hölder regularity gain of $\partial$ equation in C3," Purdue University, West Lafayette, IN, 2011. 


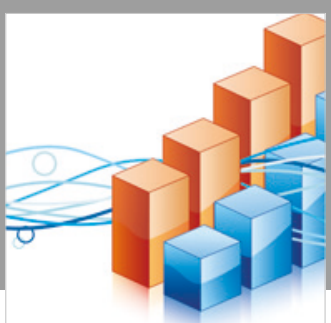

Advances in

Operations Research

\section{-n-m}
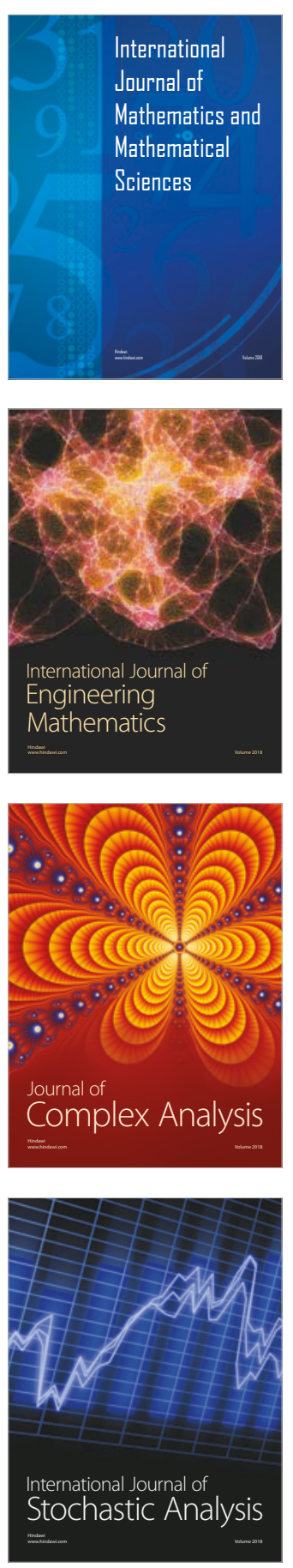
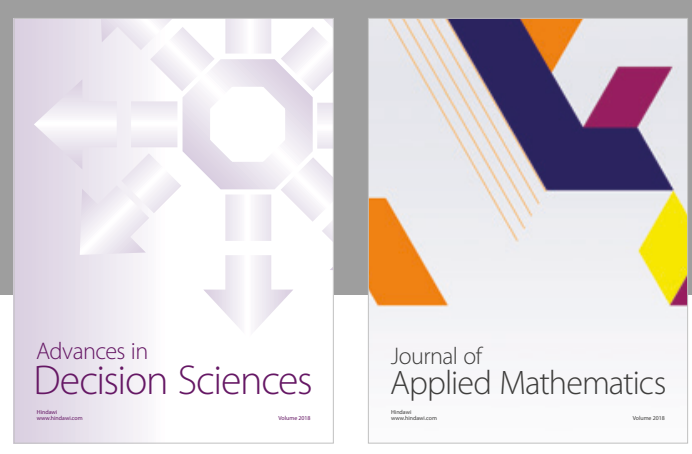

Journal of

Applied Mathematics
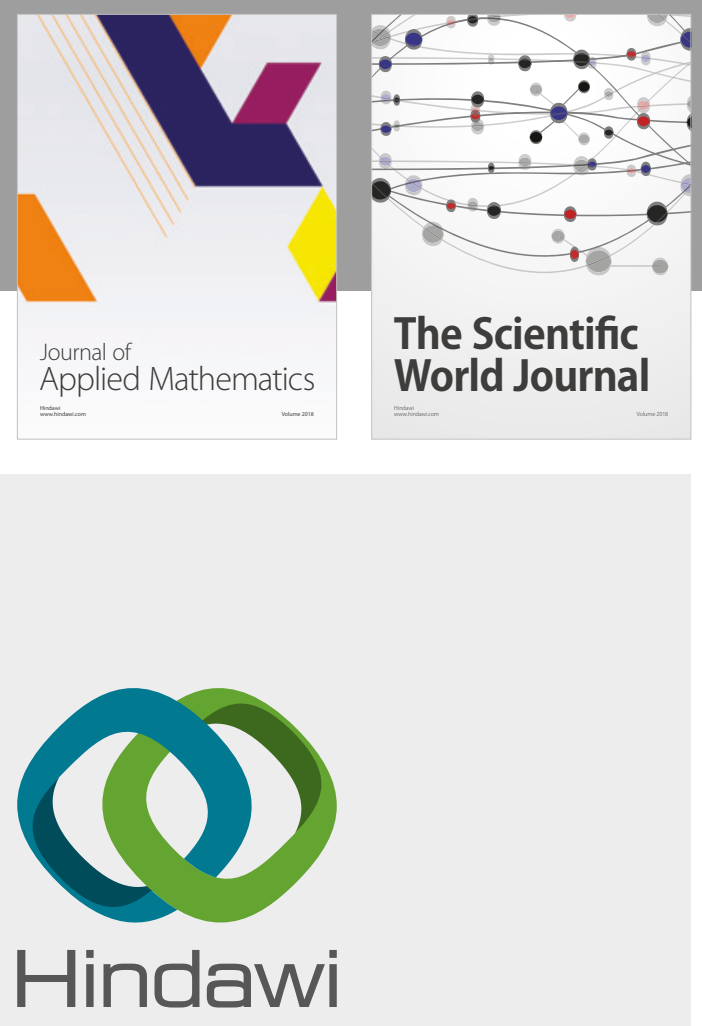

Submit your manuscripts at

www.hindawi.com

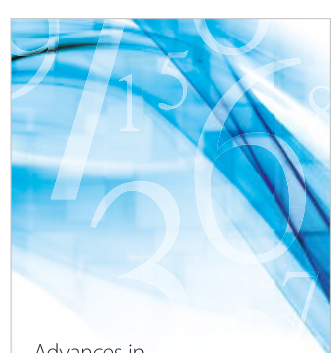

Advances in
Numerical Analysis
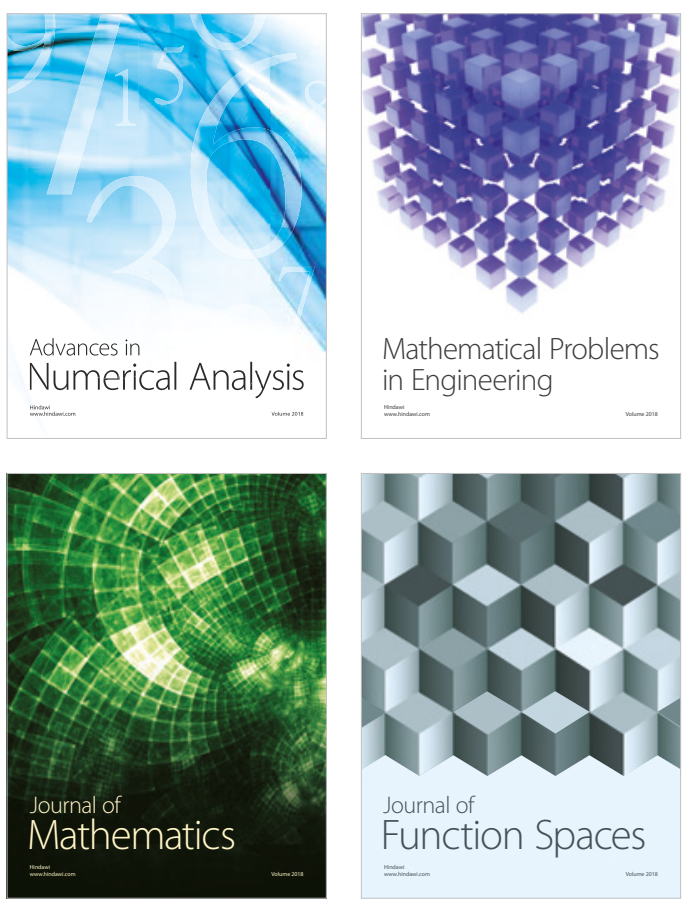

Mathematical Problems in Engineering

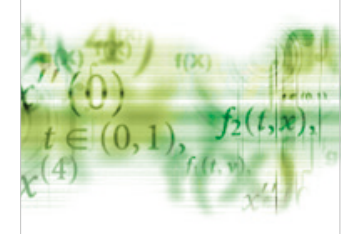

International Journal of

Differential Equations

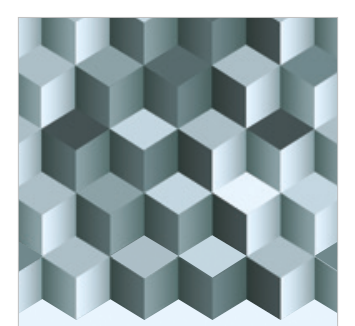

Journal of

Function Spaces

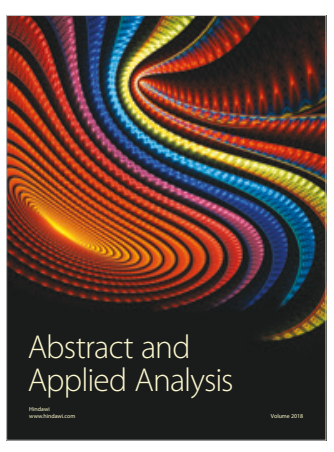

The Scientific

World Journal

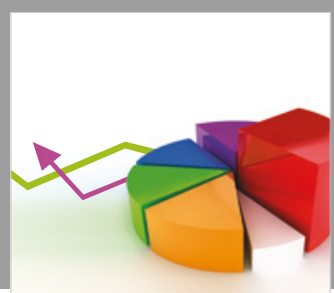

Journal of

Probability and Statistics
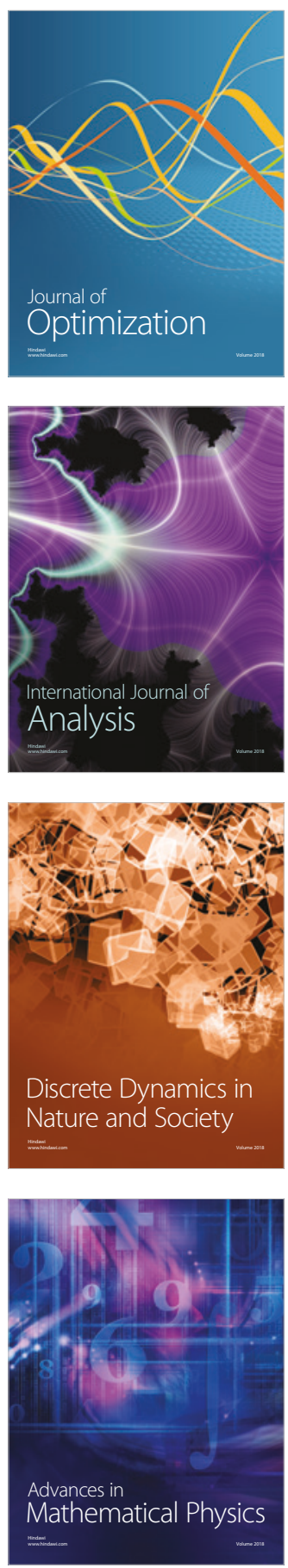